\title{
Multi-Poisson Approach to the Painlevé Equations: from the Isospectral Deformation to the Isomonodromic Deformation
}

Hayato CHIBA

Institute of Mathematics for Industry, Kyushu University, Fukuoka, 819-0395, Japan

E-mail: chiba@imi.kyushu-u.ac.jp

Received October 11, 2016, in final form April 11, 2017; Published online April 15, 2017

https://doi.org/10.3842/SIGMA.2017.025

\begin{abstract}
A multi-Poisson structure on a Lie algebra $\mathfrak{g}$ provides a systematic way to construct completely integrable Hamiltonian systems on $\mathfrak{g}$ expressed in Lax form $\partial X_{\lambda} / \partial t=$ $\left[X_{\lambda}, A_{\lambda}\right]$ in the sense of the isospectral deformation, where $X_{\lambda}, A_{\lambda} \in \mathfrak{g}$ depend rationally on the indeterminate $\lambda$ called the spectral parameter. In this paper, a method for modifying the isospectral deformation equation to the Lax equation $\partial X_{\lambda} / \partial t=\left[X_{\lambda}, A_{\lambda}\right]+\partial A_{\lambda} / \partial \lambda$ in the sense of the isomonodromic deformation, which exhibits the Painlevé property, is proposed. This method gives a few new Painlevé systems of dimension four.
\end{abstract}

Key words: Painlevé equations; Lax equations; multi-Poisson structure

2010 Mathematics Subject Classification: 34M35; 34M45; 34M55

\section{Introduction}

A differential equation defined on a complex region is said to have the Painlevé property if any movable singularity of any solution is a pole. Painlevé and his group classified second order ODEs having the Painlevé property and found new six differential equations called the Painlevé equations. Nowadays, it is known that they are written in Hamiltonian forms

$$
\left(\mathrm{P}_{\mathrm{J}}\right): \frac{d q}{d t}=\frac{\partial H_{J}}{\partial p}, \quad \frac{d p}{d t}=-\frac{\partial H_{J}}{\partial q}, \quad J=\mathrm{I}, \ldots, \mathrm{VI} .
$$

Among six Painlevé equations, the Hamiltonian functions of the first, second and fourth Painlevé equations are polynomials in both of the independent variable $t$ and the dependent variables $(q, p)$. They are given by

$$
\begin{aligned}
& H_{\mathrm{I}}=\frac{1}{2} p^{2}-2 q^{3}-t q, \\
& H_{\mathrm{II}}=\frac{1}{2} p^{2}-\frac{1}{2} q^{4}-\frac{1}{2} t q^{2}-\alpha q, \\
& H_{\mathrm{IV}}=-p q^{2}+p^{2} q-2 p q t-\alpha p+\beta q,
\end{aligned}
$$

respectively, where $\alpha, \beta \in \mathbb{C}$ are arbitrary parameters. Another important property of the Painlevé equations is that they are expressed as Lax equations. Let $L_{\lambda}$ and $A_{\lambda}$ be square matrices which depend rationally on the indeterminate $\lambda$ called the spectral parameter. The Painlevé equations are written in Lax form as

$$
\frac{\partial L_{\lambda}}{\partial t}=\left[L_{\lambda}, A_{\lambda}\right]+\frac{\partial A_{\lambda}}{\partial \lambda},
$$

This paper is a contribution to the Special Issue on Symmetries and Integrability of Difference Equations. The full collection is available at http://www.emis.de/journals/SIGMA/SIDE12.html 
for some choice of $L_{\lambda}$ and $A_{\lambda}$. This equation arises from the compatibility condition of the two differential systems

$$
\frac{\partial \Psi}{\partial \lambda}=L_{\lambda} \Psi, \quad \frac{\partial \Psi}{\partial t}=A_{\lambda} \Psi .
$$

Since the monodromy of the former system $\partial \Psi / \partial \lambda=L_{\lambda} \Psi$ is independent of $t$ if the equation (1.4) is satisfied, (1.4) is called the isomonodromic deformation equation.

Another type of the Lax equation is of the form

$$
\frac{\partial X_{\lambda}}{\partial t}=\left[X_{\lambda}, A_{\lambda}\right]
$$

which is called the isospectral deformation equation because the eigenvalues of the matrix $X_{\lambda}$ is independent of $t$. There are several systematic ways to construct isospectral deformation equations [1]. In particular, a Lie algebraic method have been often employed. Let $\mathfrak{g}$ be a Lie algebra. On the dual space $\mathfrak{g}^{*}$, there exists a canonical Poisson structure called the Lie-Poisson structure. If $\mathfrak{g}$ is equipped with a nondegenerate bilinear symmetric form, the Lie-Poisson structure is also defined on $\mathfrak{g}$. Let $P: T^{*} \mathfrak{g} \rightarrow T \mathfrak{g}$ be the Poisson tensor and $F: \mathfrak{g} \rightarrow \mathbb{C}$ a smooth function. Then, the vector field $P d F$ on $\mathfrak{g}$ can be expressed as the Lax equation (1.5) with some $X_{\lambda}, A_{\lambda} \in \mathfrak{g}[1]$.

It is notable that the isospectral deformation equation (1.5) is completely integrable for most examples, although the isomonodromic deformation equation (1.4) is not in general; it is believed that solutions of an isomonodromic deformation equation define new functions called the Painlevé transcendents.

In Nakamura [17], a way to obtain the isospectral deformation equation (1.5) from the isomonodromic deformation equation (1.4) by a certain scaling of the time $t$ is proposed, which is called the autonomous limit. She proved that the autonomous limits of 6-types of two dimensional Painlevé equations and 40-types of four dimensional Painlevé equations are completely integrable. Such relations of Painlevé systems with autonomous integrable systems are known between Gaudin model and Schlesinger system, and also found in [13] (Painlevé-Calogero correspondence).

The purpose in the present paper is opposite; a way to construct the isomonodromic deformation equation (1.4) from the isospectral deformation equation (1.5) will be proposed. Let $\mathfrak{g}$ be a simple Lie algebra over $\mathbb{C}$. Consider the set of $\mathfrak{g}$-valued polynomials of degree $n$

$$
\mathfrak{g}_{n}:=\left\{X_{\lambda}:=X_{0} \lambda^{n}+X_{1} \lambda^{n-1}+\cdots+X_{n} \mid X_{i} \in \mathfrak{g}\right\},
$$

with the indeterminate $\lambda$. This set $\mathfrak{g}_{n}$ is equipped with a structure of a Lie algebra by a certain Lie bracket. At first, the isospectral deformation equation (1.5) on $\mathfrak{g}_{n}$ is constructed with the aid of the bi-Poisson theory of Magri et al. [9, 14, 15, 16]. Isospectral deformation equations obtained in this method are shown to be completely integrable (Theorem 2.4). Next, we restrict the equations onto a symplectic leaf. Let $\varphi_{1}, \ldots, \varphi_{N}$ be Casimir functions of an underlying Poisson structure on $\mathfrak{g}_{n}$. A symplectic leaf $S$ is defined by the level surface of them as

$$
S:=\left\{\varphi_{i}=\alpha_{i} \text { (const) } \mid i=1, \ldots, N\right\} .
$$

Restricted on the leaf $S$, the isospectral deformation equation (1.5) becomes an integrable Hamiltonian system. Since the matrix $X_{\lambda} \in \mathfrak{g}_{n}$ depends on the parameters $\alpha:=\left(\alpha_{1}, \ldots, \alpha_{N}\right)$, it is denoted as $X_{\lambda}=X_{\lambda}(t, \alpha)$.

Now suppose that there exists a parameter, say $\alpha_{j}$, such that the following condition holds

$$
\frac{\partial X_{\lambda}}{\partial \alpha_{j}}(t, \alpha)=\frac{\partial A_{\lambda}}{\partial \lambda} .
$$


Equation (1.5) is put together with equation (1.6) to yield

$$
\frac{\partial X_{\lambda}}{\partial t}(t, \alpha)+\frac{\partial X_{\lambda}}{\partial \alpha_{j}}(t, \alpha)=\left[X_{\lambda}, A_{\lambda}\right]+\frac{\partial A_{\lambda}}{\partial \lambda} .
$$

Define the Lax matrix $L_{\lambda}$ by

$$
L_{\lambda}:=\left.X_{\lambda}(t, \alpha)\right|_{\alpha_{j}=t},
$$

where the parameter $\alpha_{j}$ satisfying the condition (1.6) is replaced by $t$. Then, the above equation is rewritten as the isomonodromic deformation equation (1.4).

Remark that the isomonodromic deformation equation (1.4) is equivalent to the zero curvature condition of the connection 1 form $L_{\lambda} d \lambda+A_{\lambda} d t$ on a vector bundle over the $(t, \lambda)$-space, while the condition (1.6) is the exactness condition of the connection 1 form $X_{\lambda} d \lambda+A_{\lambda} d \alpha_{j}$.

This method is demonstrated for the following three cases (I) $\mathfrak{g}=\mathfrak{s l}(2, \mathbb{C}), n=2$, (II) $\mathfrak{g}=$ $\mathfrak{s l}(2, \mathbb{C}), n=3$ and $(\mathrm{III}) \mathfrak{g}=\mathfrak{s o}(5, \mathbb{C}), n=1$. For the case (I), the first, second and fourth Painlevé equations (1.1), (1.2), (1.3) will be obtained in Section 3.

More generally, for $\mathfrak{g}=\mathfrak{s l}(2, \mathbb{C})$ with general $n$, it seems that one can obtain several Painlevé hierarchies of dimension $2 n-2$, including the first Painlevé hierarchy $\left(\mathrm{P}_{\mathrm{I}}\right)_{m}[11,12,18]$, the second-first Painlevé hierarchy $\left(\mathrm{P}_{\mathrm{II}-1}\right)_{m}[5,6,11,12]$, the second-second Painlevé hierarchy $\left(\mathrm{P}_{\mathrm{II}-2}\right)_{m}$ and the fourth Painlevé hierarchy $\left(\mathrm{P}_{\mathrm{IV}}\right)_{m}[10,11]$. They are $2 m$-dimensional Hamiltonian PDEs of the form $(m=n-1)$

$$
\begin{aligned}
& \frac{\partial q_{j}}{\partial t_{i}}=\frac{\partial H_{i}}{\partial p_{j}}, \quad \frac{\partial p_{j}}{\partial t_{i}}=-\frac{\partial H_{i}}{\partial q_{j}}, \quad j=1, \ldots, m, \quad i=1, \ldots, m, \\
& H_{i}=H_{i}\left(q_{1}, \ldots, q_{m}, p_{1}, \ldots, p_{m}, t_{1}, \ldots, t_{m}\right)
\end{aligned}
$$

consisting of $m$ Hamiltonians $H_{1}, \ldots, H_{m}$ with $m$ independent variables $t_{1}, \ldots, t_{m}$. When $m=1$ (the case $(\mathrm{I})),\left(\mathrm{P}_{\mathrm{I}}\right)_{1}$ and $\left(\mathrm{P}_{\mathrm{IV}}\right)_{1}$ are reduced to the first and fourth Painlevé equations, respectively. Both of $\left(\mathrm{P}_{\mathrm{II}-1}\right)_{1}$ and $\left(\mathrm{P}_{\mathrm{II}-2}\right)_{1}$ coincide with the second Painlevé equation, while they are different systems for $m \geq 2$. When $m=2$ (the case (II)), Hamiltonians of $\left(\mathrm{P}_{\mathrm{I}}\right)_{2},\left(\mathrm{P}_{\mathrm{II}-1}\right)_{2},\left(\mathrm{P}_{\mathrm{II}-2}\right)_{2}$ and $\left(\mathrm{P}_{\mathrm{IV}}\right)_{2}$ are given by

$$
\begin{array}{ll}
\left(\mathrm{P}_{\mathrm{I}}\right)_{2} & \left\{\begin{array}{r}
H_{1}=2 p_{2} p_{1}+3 p_{2}^{2} q_{1}+q_{1}^{4}-q_{1}^{2} q_{2}-q_{2}^{2}-t_{1} q_{1}+t_{2}\left(q_{1}^{2}-q_{2}\right), \\
H_{2}=p_{1}^{2}+2 p_{2} p_{1} q_{1}-q_{1}^{5}+p_{2}^{2} q_{2}+3 q_{1}^{3} q_{2}-2 q_{1} q_{2}^{2} \\
\quad+t_{1}\left(q_{1}^{2}-q_{2}\right)+t_{2}\left(t_{2} q_{1}+q_{1} q_{2}-p_{2}^{2}\right),
\end{array}\right. \\
\left(\mathrm { P } _ { \mathrm { II } - 1 ) _ { 2 } } \quad \left\{\begin{array}{r}
H_{1}=2 p_{1} p_{2}-p_{2}^{3}-p_{1} q_{1}^{2}+q_{2}^{2}-t_{1} p_{2}+t_{2} p_{1}+2 \alpha q_{1}, \\
H_{2}=-p_{1}^{2}+p_{1} p_{2}^{2}+p_{1} p_{2} q_{1}^{2}+2 p_{1} q_{1} q_{2} \\
+t_{1} p_{1}+t_{2}\left(t_{2} p_{1}-p_{1} q_{1}^{2}+p_{1} p_{2}\right)-\alpha\left(2 p_{2} q_{1}+2 q_{2}+2 t_{2} q_{1}\right),
\end{array}\right.\right. \\
\left(\mathrm { P } _ { \mathrm { II } - 2 ) _ { 2 } } \left\{\begin{array}{r}
H_{1}=p_{1} p_{2}-p_{1} q_{1}^{2}-2 p_{1} q_{2}+p_{2} q_{1} q_{2}+q_{1} q_{2}^{2}+q_{2} t_{1}+t_{2}\left(q_{1} q_{2}-p_{1}\right)+\alpha q_{1}, \\
H_{2}=p_{1}^{2}-p_{1} p_{2} q_{1}+p_{2}^{2} q_{2}-2 p_{1} q_{1} q_{2}-p_{2} q_{2}^{2}+q_{1}^{2} q_{2}^{2} \\
+t_{1}\left(q_{1} q_{2}-p_{1}\right)-t_{2}\left(p_{1} q_{1}+q_{2}^{2}+q_{2} t_{2}\right)+\alpha p_{2},
\end{array}\right.\right. \\
\left(\mathrm { P } _ { \mathrm { IV } ) _ { 2 } } \left\{\begin{array}{r}
H_{1}=p_{1}^{2}+p_{1} p_{2}-p_{1} q_{1}^{2}+p_{2} q_{1} q_{2}-p_{2} q_{2}^{2}-t_{1} p_{1}+t_{2} p_{2} q_{2}+\alpha q_{2}+\beta q_{1}, \\
H_{2}=p_{1} p_{2} q_{1}-2 p_{1} p_{2} q_{2}-p_{2}^{2} q_{2}+p_{2} q_{1} q_{2}^{2} \\
\quad+p_{2} q_{2} t_{1}+t_{2}\left(p_{1} p_{2}-p_{2} q_{2}^{2}+p_{2} q_{2} t_{2}\right)+\left(p_{1}-q_{1} q_{2}+q_{2} t_{2}\right) \alpha-\beta p_{2},
\end{array}\right.\right.
\end{array}
$$

respectively, with arbitrary parameters $\alpha, \beta \in \mathbb{C}$. These systems will be obtained from the case (II) $\mathfrak{g}=\mathfrak{s l}(2, \mathbb{C}), n=3$ in Section 4 . In our method, such Hamiltonian PDEs are obtained if there are several Hamiltonian systems written in Lax form (1.5), and if there are several parameters satisfying (1.6); such parameters will be replaced by distinct times $t_{1}, t_{2}, \ldots$ 
We will find other 4-dimensional Painlevé systems with Hamiltonian functions

$$
\begin{aligned}
H_{(1,1,2,0)}= & -p_{1}^{2} q_{1}-2 p_{1} q_{1}^{2}+2 p_{1} q_{2}-2 p_{1} p_{2} q_{2}-2 p_{2} q_{1} q_{2} \\
& +\left(2 p_{1} q_{1}+2 p_{2} q_{2}\right) t+\left(2 \alpha_{2}+2 \beta_{2}\right) q_{1}+2 \beta_{2} p_{1}+2 \beta_{3} p_{2}, \\
H_{(-1,4,1,2)}= & p_{1}-p_{2}^{2}-2 p_{1} q_{1} q_{2}-p_{2} q_{2}^{2}+2 \beta_{3} q_{2}+2 \beta_{5} q_{1}+p_{2} t, \\
H_{\text {Cosgrove }}= & -4 p_{1} p_{2}-2 p_{2}^{2} q_{1}-\frac{73}{128} q_{1}^{4}+\frac{11}{8} q_{1}^{2} q_{2}-\frac{1}{2} q_{2}^{2}-q_{1} t-\frac{\alpha_{2}}{48}\left(q_{1}+\frac{\alpha_{2}}{6}\right) q_{1}^{2},
\end{aligned}
$$

where $\alpha_{i}, \beta_{i} \in \mathbb{C}$ are arbitrary parameters (the subscripts for parameters are related to the weighted degrees so that the Hamiltonian functions become quasihomogeneous, see below). The first two systems will be also obtained from the case (II). As far as the author knows, these systems have not appeared in the literature. The last one $H_{\text {Cosgrove }}$ will be obtained from the case (III) $\mathfrak{g}=\mathfrak{s o}(5, \mathbb{C}), n=1$ in Section 5 . If we rewrite the system as a fourth order single equation of $q_{1}=y$, we obtain

$$
y^{\prime \prime \prime \prime}=18 y y^{\prime \prime}+9\left(y^{\prime}\right)^{2}-24 y^{3}+16 t+\alpha y\left(y+\frac{1}{9} \alpha\right) .
$$

This equation was given in [7], denoted by F-VI, without a proof that it has the Painlevé property. Since this system is obtained as the isomonodromic deformation equation in this paper, this equation actually enjoys the Painlevé property. In [7] it is conjectured that this equation defines a new Painlevé transcendent (i.e., it is not reduced to known equations). Another expression of the Hamiltonian function of the same system is

$$
\widetilde{H}_{\text {Cosgrove }}=2 p_{1} p_{2}-\frac{18}{13} p_{2}^{2} q_{1}-\frac{2}{169} q_{1}^{4}-\frac{180}{13} q_{1}^{2} q_{2}+6 q_{2}^{2}-8 q_{1} t+\frac{8}{9} \alpha_{2} q_{1}^{3}+\frac{8}{27} \alpha_{2}^{2} q_{1}^{2}
$$

The corresponding Hamiltonian system is also reduced to (1.14).

Note that all of the Hamiltonian functions above are polynomials in both of the independent variables and the dependent variables. Furthermore, they are semi-quasihomogeneous functions. In general, a polynomial $H\left(x_{1}, \ldots, x_{n}\right)$ is called a quasihomogeneous polynomial if there are integers $a_{1}, \ldots, a_{n}$ and $h$ such that

$$
H\left(\lambda^{a_{1}} x_{1}, \ldots, \lambda^{a_{n}} x_{n}\right)=\lambda^{h} H\left(x_{1}, \ldots, x_{n}\right)
$$

for any $\lambda \in \mathbb{C}$. A polynomial $H$ is called a semi-quasihomogeneous if $H$ is decomposed into two polynomials as $H=H^{P}+H^{N}$, where $H^{P}$ satisfies (1.16) and $H^{N}$ satisfies

$$
H^{N}\left(\lambda^{a_{1}} x_{1}, \ldots, \lambda^{a_{n}} x_{n}\right) \sim o\left(\lambda^{h}\right), \quad|\lambda| \rightarrow \infty .
$$

The integer $\operatorname{wdeg}(H):=h$ is called the weighted degree of $H$ with respect to the weight $\operatorname{wdeg}\left(x_{1}, \ldots, x_{n}\right):=\left(a_{1}, \ldots, a_{n}\right)$. For example, if we define degrees of variables by $\operatorname{wdeg}(q, p, t)=$ $(2,3,4)$ for $H_{\mathrm{I}}, \operatorname{wdeg}(q, p, t)=(1,2,2)$ for $H_{\mathrm{II}}$ and $\operatorname{wdeg}(q, p, t)=(1,1,1)$ for $H_{\mathrm{IV}}$, then Hamiltonian functions have the weighted degrees 6, 4 and 3, respectively (Table 1). The weights for four dimensional systems above are shown in Table 2. In this paper, these weights are naturally obtained from a suitable definition of weights of entries of a matrix $X_{\lambda} \in \mathfrak{g}_{n}$ and the spectral parameter $\lambda$. In particular, the weights of the Hamiltonian functions are closely related to the exponents of simple Lie algebras because the Hamiltonian functions are essentially Ad-invariant polynomials of simple Lie algebras. See $[2,3,4]$ for the detailed study of the weights of the Painlevé equations. 
Table 1. Weights for two dimensional Painlevé equations.

\begin{tabular}{|c||c|c|}
\hline & $\operatorname{wdeg}(q, p, t)$ & $\operatorname{wdeg}(H)$ \\
\hline \hline $\mathrm{P}_{\mathrm{I}}$ & $(2,3,4)$ & 6 \\
\hline $\mathrm{P}_{\mathrm{II}}$ & $(1,2,2)$ & 4 \\
\hline $\mathrm{P}_{\mathrm{IV}}$ & $(1,1,1)$ & 3 \\
\hline
\end{tabular}

Table 2. Weights for four dimensional Painlevé equations.

\begin{tabular}{|c||c|c|c|}
\hline & $\operatorname{wdeg}\left(q_{1}, p_{1}, q_{2}, p_{2}\right)$ & $\operatorname{wdeg}\left(t_{1}, t_{2}\right)$ & $\operatorname{wdeg}\left(H_{1}, H_{2}\right)$ \\
\hline \hline$\left(\mathrm{P}_{\mathrm{I}}\right)_{2}$ & $(2,5,4,3)$ & 6,4 & 8,10 \\
\hline$\left(\mathrm{P}_{\mathrm{II}-1}\right)_{2}$ & $(1,4,3,2)$ & 4,2 & 6,8 \\
\hline$\left(\mathrm{P}_{\mathrm{II}-2}\right)_{2}$ & $(1,3,2,2)$ & 3,2 & 5,6 \\
\hline$\left(\mathrm{P}_{\mathrm{IV}}\right)_{2}$ & $(1,2,1,2)$ & 2,1 & 4,5 \\
\hline$H_{(1,1,2,0)}$ & $(1,1,2,0)$ & 1 & 3 \\
\hline$H_{(-1,4,1,2)}$ & $(-1,4,1,2)$ & 2 & 4 \\
\hline$H_{\text {Cosgrove }}$ & $(2,5,4,3)$ & 6 & 8 \\
\hline
\end{tabular}

\section{Settings}

\subsection{Lie-Poisson structure on $\mathfrak{g}_{n}$}

We define a multi-Poisson structure on a certain Lie algebra following Magri et al. [9, 14, 15, 16]. Let $(\mathfrak{g},[\cdot, \cdot])$ be a simple Lie algebra over $\mathbb{C}$. Consider the set of $\mathfrak{g}$-valued polynomials of degree $n$

$$
\mathfrak{g}_{n}:=\left\{X_{\lambda}:=X_{0} \lambda^{n}+X_{1} \lambda^{n-1}+\cdots+X_{n} \mid X_{i} \in \mathfrak{g}\right\},
$$

with the indeterminate $\lambda$. The bracket defined by

$$
\begin{aligned}
{\left[X_{\lambda}, Y_{\lambda}\right]_{n}:=} & {\left[X_{n}, Y_{n}\right]+\lambda\left(\left[X_{n}, Y_{n-1}\right]+\left[X_{n-1}, Y_{n}\right]\right)+\cdots } \\
& +\lambda^{n}\left(\left[X_{0}, Y_{n}\right]+\left[X_{1}, Y_{n-1}\right]+\cdots+\left[X_{n}, Y_{0}\right]\right)
\end{aligned}
$$

introduces the structure of a Lie algebra on $\mathfrak{g}_{n}$. Note that $\left[X_{\lambda}, Y_{\lambda}\right]_{n}$ coincides with $\left[X_{\lambda}, Y_{\lambda}\right]$ expanded in $\lambda$ and truncated at degree $n$.

It is known that the dual space $\mathfrak{g}^{*}$ of any Lie algebra $\mathfrak{g}$ is equipped with a canonical Poisson structure called the Lie-Poisson structure. If a nondegenerate symmetric bilinear form $\eta: \mathfrak{g} \times \mathfrak{g} \rightarrow \mathbb{C}$ is defined on $\mathfrak{g}$, it induces a Lie-Poisson structure on $\mathfrak{g}$. For functions $F, G: \mathfrak{g} \rightarrow \mathbb{C}$, the Poisson bracket on $\mathfrak{g}$ is defined by $\{F, G\}(X)=\eta(X,[\nabla F(X), \nabla G(X)])$, where $\nabla F(X) \in \mathfrak{g}$ is defined through $(d F)_{X}(Y)=\eta(\nabla F(X), Y)$. To give the Lie-Poisson structure on $\mathfrak{g}_{n}$, we define a nondegenerate symmetric bilinear form $\eta$ on $\mathfrak{g}_{n}$ by

$$
\eta\left(X_{\lambda}, Y_{\lambda}\right):=\sum_{i=0}^{n} \operatorname{Tr}\left(X_{i} Y_{n-i}\right),
$$

by which $\mathfrak{g}_{n}$ is identified with its dual. For a smooth function $F: \mathfrak{g}_{n} \rightarrow \mathbb{C}$, define the gradient $\nabla F \in \mathfrak{g}_{n}$ through $(d F)\left(Y_{\lambda}\right)=\eta\left(\nabla F, Y_{\lambda}\right)$, and define $\nabla_{i} F \in \mathfrak{g}$ by

$$
\nabla F=\left(\nabla_{n} F\right) \lambda^{n}+\left(\nabla_{n-1} F\right) \lambda^{n-1}+\cdots+\nabla_{0} F .
$$

Using them, the Lie-Poisson bracket on $\mathfrak{g}_{n}$ is given by

$$
\{F, G\}_{0}:=\eta\left(X_{\lambda},[\nabla F, \nabla G]_{n}\right)
$$




$$
\begin{aligned}
= & \operatorname{Tr}\left(X_{0} \cdot\left[\nabla_{0} F, \nabla_{0} G\right]\right)+\operatorname{Tr}\left(X_{1} \cdot\left(\left[\nabla_{0} F, \nabla_{1} G\right]+\left[\nabla_{1} F, \nabla_{0} G\right]\right)\right)+\cdots \\
& +\operatorname{Tr}\left(X_{n} \cdot\left(\left[\nabla_{0} F, \nabla_{n} G\right]+\cdots+\left[\nabla_{n} F, \nabla_{0} G\right]\right)\right) \\
= & -\operatorname{Tr}\left(\nabla_{0} F \cdot\left(\left[X_{0}, \nabla_{0} G\right]+\left[X_{1}, \nabla_{1} G\right]+\cdots+\left[X_{n}, \nabla_{n} G\right]\right)\right)-\cdots \\
& -\operatorname{Tr}\left(\nabla_{n-1} F \cdot\left(\left[X_{n-1}, \nabla_{0} G\right]+\left[X_{n}, \nabla_{1} G\right]\right)\right)-\operatorname{Tr}\left(\nabla_{n} F \cdot\left[X_{n}, \nabla_{0} G\right]\right) .
\end{aligned}
$$

The Poisson tensor (bivector) $P_{0}: T^{*} \mathfrak{g}_{n} \rightarrow T \mathfrak{g}_{n}$ is defined so that

$$
\{F, G\}_{0}=d F\left(P_{0} d G\right)=\eta\left(\nabla F, P_{0} d G\right)=\sum_{i=0}^{n} \operatorname{Tr}\left(\nabla_{i} F \cdot\left(P_{0} d G\right)_{i}\right)
$$

This implies

$$
\begin{aligned}
& -\left(P_{0} d G\right)_{0}=\left[X_{0}, \nabla_{0} G\right]+\left[X_{1}, \nabla_{1} G\right]+\cdots+\left[X_{n}, \nabla_{n} G\right], \\
& -\left(P_{0} d G\right)_{n-1}=\left[X_{n-1}, \nabla_{0} G\right]+\left[X_{n}, \nabla_{1} G\right] \text {, } \\
& -\left(P_{0} d G\right)_{n}=\left[X_{n}, \nabla_{0} G\right] \text {. }
\end{aligned}
$$

The following expression is useful

$$
\begin{aligned}
P_{0}: d G \mapsto- & \left(\begin{array}{cccc}
{\left[X_{0}, \cdot\right]} & {\left[X_{1}, \cdot\right]} & \ldots & {\left[X_{n}, \cdot\right]} \\
\vdots & & . \cdot & \\
{\left[X_{n-1}, \cdot\right]} & {\left[X_{n}, \cdot\right]} & \\
{\left[X_{n}, \cdot\right]} &
\end{array}\right)\left(\begin{array}{c}
\nabla_{0} G \\
\vdots \\
\nabla_{n-1} G \\
\nabla_{n} G
\end{array}\right) \\
= & \left(\begin{array}{c}
{\left[\nabla_{0} G, X_{0}\right]+\left[\nabla_{1} G, X_{1}\right]+\cdots+\left[\nabla_{n} G, X_{n}\right]} \\
\vdots \\
{\left[\nabla_{0} G, X_{n-1}\right]+\left[\nabla_{1} G, X_{n}\right]} \\
{\left[\nabla_{0} G, X_{n}\right]}
\end{array}\right) .
\end{aligned}
$$

It is also represented as a matrix as follows. Let $A=A(X)$ be a representation matrix of the mapping

$$
T^{*} \mathfrak{g}(\simeq \mathfrak{g}) \rightarrow \mathfrak{g}, \quad d G \mapsto[X, \nabla G], \quad G: \mathfrak{g} \rightarrow \mathbb{C}, \quad X \in \mathfrak{g}
$$

with respect to some coordinates on $\mathfrak{g}$ (here $\nabla G$ is the gradient on $\mathfrak{g}$ ). By the definition, $-A$ is a Poisson tensor of the Lie-Poisson structure on $\mathfrak{g}$. Since $A(X)$ is linear in $X, A\left(X_{\lambda}\right)$ is expanded as $A\left(X_{\lambda}\right)=\lambda^{n} A\left(X_{0}\right)+\lambda^{n-1} A\left(X_{1}\right)+\cdots+A\left(X_{n}\right)$. Putting $A\left(X_{j}\right)=A_{j}, P_{0}$ is represented as an $(n+1) \operatorname{dim}(\mathfrak{g}) \times(n+1) \operatorname{dim}(\mathfrak{g})$ matrix

$$
P_{0}=-\left(\begin{array}{cccc}
A_{0} & \ldots & A_{n-1} & A_{n} \\
A_{1} & \ldots & A_{n} & \\
\vdots & . & & \\
A_{n} & & &
\end{array}\right) .
$$

In what follows, suppose $\operatorname{dim}(\mathfrak{g})=d, \operatorname{rank}(\mathfrak{g})=h$ and let $m_{1}, \ldots, m_{h}$ be exponents of $\mathfrak{g}$. Let $\left(y_{1}, \ldots, y_{d}\right)$ be coordinates on $\mathfrak{g}$. It is known that the Casimir functions of the Lie-Poisson structure on $\mathfrak{g}$ (i.e., a function $\varphi$ satisfying $\{F, \varphi\}=0$ for any $F: \mathfrak{g} \rightarrow \mathbb{C}$ ) are the Ad-invariant polynomials denoted by $\varphi_{i}\left(y_{1}, \ldots, y_{d}\right), i=1, \ldots, h$, and they satisfy $\operatorname{deg}\left(\varphi_{i}\right)=m_{i}+1$.

Let $x_{j}:=\left(x_{j, 1}, \ldots, x_{j, d}\right)$ be coordinates on the $j$-th copy of $\mathfrak{g}$ (coordinate expression for $X_{j}$ ) and $\left(x_{0}, \ldots, x_{n}\right)$ coordinates on $\mathfrak{g}_{n}$. We define the weighted degrees of variables to be

$$
\operatorname{wdeg}\left(x_{j}\right)=\operatorname{wdeg}\left(x_{j, \alpha}\right)=j, \quad \operatorname{wdeg}(\lambda)=1 .
$$


Then, $X_{\lambda}$ is quasihomogeneous (homogeneous in the weighted sense) of $\operatorname{wdeg}\left(X_{\lambda}\right)=n$. Substituting $y_{\alpha}=x_{0, \alpha} \lambda^{n}+x_{1, \alpha} \lambda^{n-1}+\cdots+x_{n, \alpha}$ into $\varphi_{i}\left(y_{1}, \ldots, y_{d}\right)$ and expanding it in $\lambda$ provide

$$
\begin{aligned}
\varphi_{i}\left(y_{1}, \ldots, y_{d}\right)= & \varphi_{i, 0}\left(x_{0}, \ldots, x_{n}\right) \lambda^{\left(m_{i}+1\right) n}+\varphi_{i, 1}\left(x_{0}, \ldots, x_{n}\right) \lambda^{\left(m_{i}+1\right) n-1}+\cdots \\
& +\varphi_{i,\left(m_{i}+1\right) n}\left(x_{0}, \ldots, x_{n}\right), \quad i=1, \ldots, h,
\end{aligned}
$$

which defines polynomials $\varphi_{i, j}$ on $\mathfrak{g}_{n}$ satisfying

$$
\operatorname{deg}\left(\varphi_{i, j}\right)=m_{i}+1, \quad \operatorname{wdeg}\left(\varphi_{i, j}\right)=j .
$$

\section{Proposition 2.1.}

(i) $\varphi_{i, j}$ depends only on $\left(x_{0}, \ldots, x_{j}\right)$ for $0 \leq j \leq n-1$.

(ii) $\varphi_{i, j}\left(x_{0}, x_{1}, \ldots, x_{n}\right)=\varphi_{i,\left(m_{i}+1\right) n-j}\left(x_{n}, \ldots, x_{1}, x_{0}\right)$.

(iii) For each $i, j, \alpha$, the derivative $\partial \varphi_{i, j+k} / \partial x_{k, \alpha}$ is independent of $k=0, \ldots, n$.

(iv) For each $i, j$, the gradient $\nabla_{k} \varphi_{i, j+k}$ is independent of $k=0, \ldots, n$.

(v) For each $i, j, k$, the equality

$$
\sum_{l=0}^{n} A_{l} \frac{\partial \varphi_{i, j+k-l}}{\partial x_{k}}=\sum_{l=0}^{n} A_{l} \frac{\partial \varphi_{i, j-l}}{\partial x_{0}}=0
$$

holds.

(vi) The Casimir functions of the Lie-Poisson structure $P_{0}$ on $\mathfrak{g}_{n}$ are

$$
\varphi_{i,\left(m_{i}+1\right) n-j,} i=1, \ldots, h, \quad j=0, \ldots, n .
$$

Proof. (i) and (ii) follow from the definition of $\varphi_{i, j}$.

(iii) For $y_{\alpha}=\sum_{k=0}^{n} \lambda^{n-k} x_{k, \alpha}$, we have

$$
\begin{aligned}
\frac{\partial \varphi_{i}}{\partial y_{\alpha}} & =\frac{\partial x_{k, \alpha}}{\partial y_{\alpha}} \frac{\partial}{\partial x_{k, \alpha}} \sum_{j=0}^{\left(m_{i}+1\right) n} \lambda^{\left(m_{i}+1\right) n-j} \varphi_{i, j} \\
& =\sum_{j=0}^{\left(m_{i}+1\right) n} \lambda^{m_{i} n-j+k} \frac{\partial \varphi_{i, j}}{\partial x_{k, \alpha}}=\sum_{j=k}^{m_{i} n+k} \lambda^{m_{i} n-j+k} \frac{\partial \varphi_{i, j}}{\partial x_{k, \alpha}} .
\end{aligned}
$$

For the last equality, we used part (i) combined with part (ii). Thus we obtain

$$
\frac{\partial \varphi_{i}}{\partial y_{\alpha}}=\sum_{j=0}^{m_{i} n} \lambda^{m_{i} n-j} \frac{\partial \varphi_{i, j+k}}{\partial x_{k, \alpha}} .
$$

Since the left hand side is independent of $k$, so is each coefficient of $\lambda^{m_{i} n-j}$ in the right hand side. Part (iv) immediately follows from (iii).

(v) The first equality is a consequence of part (iii). Since $\varphi_{i}(y)$ is a Casimir function of the Lie-Poisson structure on $\mathfrak{g}, \operatorname{Ad} \varphi_{i}=0$, where $A$ is a matrix defined before. Substituting $y=\sum_{k=0}^{n} \lambda^{n-k} x_{k}$ yields

$$
\begin{aligned}
0 & =A \frac{\partial \varphi_{i}}{\partial y}=\left(\lambda^{n} A_{0}+\lambda^{n-1} A_{1}+\cdots+A_{n}\right) \sum_{j=0}^{m_{i} n} \lambda^{m_{i} n-j} \frac{\partial \varphi_{i, j+k}}{\partial x_{k}} \\
& =\sum_{j, l} \lambda^{m_{i} n-j+n-l} A_{l} \frac{\partial \varphi_{i, j+k}}{\partial x_{k}}=\sum_{j=l}^{m_{i} n+l} \lambda^{m_{i} n+n-j} \sum_{l=0}^{n} A_{l} \frac{\partial \varphi_{i, j+k-l}}{\partial x_{k}} .
\end{aligned}
$$

This proves the second equality of $(\mathrm{v})$. 
To prove (vi), it is sufficient to show

$$
\left(\begin{array}{cccc}
A_{0} & \ldots & A_{n-1} & A_{n} \\
A_{1} & \ldots & A_{n} & \\
\vdots & . \cdot & & \\
A_{n} & & &
\end{array}\right)\left(\begin{array}{c}
\partial \varphi_{i,\left(m_{i}+1\right) n-j} / \partial x_{0} \\
\partial \varphi_{i,\left(m_{i}+1\right) n-j} / \partial x_{1} \\
\vdots \\
\partial \varphi_{i,\left(m_{i}+1\right) n-j} / \partial x_{n}
\end{array}\right)=0
$$

for $j=0, \ldots, n$. This is verified with the aid of part (v).

Example 2.2. For $\mathfrak{g}=\mathfrak{s l}(2, \mathbb{C})$, we have $d=3, h=1$ and $m_{i}=m_{1}=1$. Denote a general element $X_{\lambda} \in \mathfrak{g}_{n}$ as

$$
\begin{aligned}
X_{\lambda} & =\lambda^{n} X_{0}+\lambda^{n-1} X_{1}+\cdots+X_{n} \\
& =\lambda^{n}\left(\begin{array}{cc}
u_{0} & v_{0} \\
w_{0} & -u_{0}
\end{array}\right)+\lambda^{n-1}\left(\begin{array}{cc}
u_{1} & v_{1} \\
w_{1} & -u_{1}
\end{array}\right)+\cdots+\left(\begin{array}{cc}
u_{n} & v_{n} \\
w_{n} & -u_{n}
\end{array}\right) .
\end{aligned}
$$

Let $\left(u_{j}, v_{j}, w_{j}\right)$ be coordinates on the $j$-th copy of $\mathfrak{g}$ and $\left(u_{0}, v_{0}, w_{0}, \ldots, u_{n}, v_{n}, w_{n}\right)$ coordinates on $\mathfrak{g}_{n}$. Then,

$$
\nabla_{j} F=\left(\begin{array}{cc}
\frac{1}{2} \frac{\partial F}{\partial u_{j}} & \frac{\partial F}{\partial w_{j}} \\
\frac{\partial F}{\partial v_{j}} & -\frac{1}{2} \frac{\partial F}{\partial u_{j}}
\end{array}\right), \quad A_{j}=\left(\begin{array}{ccc}
0 & v_{j} & -w_{j} \\
-v_{j} & 0 & 2 u_{j} \\
w_{j} & -2 u_{j} & 0
\end{array}\right) .
$$

The Casimir function on $\mathfrak{g}$ is given by $\varphi_{i}=\varphi=u^{2}+v w$. Then, the functions $\varphi_{i, j}=\varphi_{j}$ are defined by expanding

$$
\left(\lambda^{n} u_{0}+\cdots+u_{n}\right)^{2}+\left(\lambda^{n} v_{0}+\cdots+v_{n}\right)\left(\lambda^{n} w_{0}+\cdots+w_{n}\right)
$$

in $\lambda$. This gives

$$
\varphi_{j}=\sum_{k+l=j}\left(u_{k} u_{l}+v_{k} w_{l}\right), \quad j=0, \ldots, 2 n
$$

Note that they are coefficients of $-\operatorname{det} X_{\lambda}$. The Casimir functions of $\mathfrak{g}_{n}$ are given by $\varphi_{j}$ for $j=n, \ldots, 2 n$.

\section{$2.2 \quad$ Multi-Poisson structure on $\mathfrak{g}_{n}^{0}$}

In general, a manifold $M$ is called a bi-Poisson manifold if

(i) there are two Poisson brackets $\{,\}_{0}$ and $\{,\}_{1}$, and

(ii) the linear combination $\{,\}_{0}+t\{,\}_{1}$ is also a Poisson bracket for any $t \in \mathbb{C}$.

See $[9,14,15,16]$ for applications of bi-Poisson manifolds to integrable systems. Here, we introduce a bi-Poisson structure on $\mathfrak{g}_{n}$ following [14]. The shift operator $X_{\lambda} \mapsto X_{\lambda+t}$ defines an automorphism of $\mathfrak{g}_{n}$ with a parameter $t \in \mathbb{C}$. It induces a deformation, denoted by $\{,\}_{t}$, of the Lie-Poisson bracket $\{,\}_{0}$. Let

$$
\{,\}_{t}=\{,\}_{0}+t\{,\}_{1}+\cdots+t^{n+1}\{,\}_{n+1}+\cdots
$$


be its expansion. Magnano and Magri [14] proved that each $\{,\}_{i}, i=0, \ldots, n+1$, and their any linear combination satisfy the axiom of a Poisson bracket. Hence, $\mathfrak{g}_{n}$ has $n+2$ compatible Poisson brackets and it becomes a multi-Poisson manifold. Their Poisson tensors are

$$
\begin{aligned}
& P_{1}=\left(\begin{array}{c|cccc}
0 & 0 & 0 & \ldots & 0 \\
\hline 0 & -A_{1} & -A_{2} & \ldots & -A_{n} \\
\vdots & \vdots & \vdots & . & \\
0 & -A_{n-1} & -A_{n} & & \\
0 & -A_{n} & & &
\end{array}\right) \text {, } \\
& P_{k+1}=\left(\begin{array}{c|ccc|ccc}
0 & 0 & \ldots & 0 & 0 & \ldots & 0 \\
\hline 0 & & & A_{0} & & & \\
\vdots & & . & \vdots & & & \\
0 & A_{0} & \ldots & A_{k-1} & & & \\
\hline 0 & & & & -A_{k+1} & \ldots & -A_{n} \\
\vdots & & & & \vdots & . & \\
0 & & & & -A_{n} &
\end{array}\right), \quad k=1, \ldots, n-1, \\
& P_{n+1}=\left(\begin{array}{c|cccc}
0 & 0 & \ldots & 0 & 0 \\
\hline 0 & & & & A_{0} \\
0 & & & A_{0} & A_{1} \\
\vdots & & . & \vdots & \vdots \\
0 & A_{0} & \ldots & A_{n-2} & A_{n-1}
\end{array}\right) .
\end{aligned}
$$

( $P_{0}$ is the same as before). Let $\mathfrak{g}_{n}^{0}$ be a submanifold of $\mathfrak{g}_{n}$ defined by $x_{0}=$ const;

$$
\mathfrak{g}_{n}^{0}:=\left\{X_{\lambda}=X_{0} \lambda^{n}+X_{1} \lambda^{n-1}+\cdots+X_{n} \mid X_{0}=\text { const }\right\} \subset \mathfrak{g}_{n} .
$$

Since the first row and column of $P_{1}, \ldots, P_{n+1}$ are zero (i.e., $x_{0}=\left(x_{0,1}, \ldots, x_{0, d}\right)$ are Casimir functions of them), the restrictions of them on $\mathfrak{g}_{n}^{0}$ define a multi-Poisson structure on $\mathfrak{g}_{n}^{0}$, whose brackets and tensors are again denoted by $\left(\{,\}_{i}, P_{i}\right)$. The tensors are given by

$$
\begin{aligned}
& P_{1}=\left(\begin{array}{cccc}
-A_{1} & -A_{2} & \ldots & -A_{n} \\
\vdots & \vdots & . & \\
-A_{n-1} & -A_{n} & & \\
-A_{n} & & &
\end{array}\right), \\
& P_{k+1}=\left(\begin{array}{ccc|ccc} 
& & A_{0} & & & \\
& . & \vdots & & & \\
A_{0} & \ldots & A_{k-1} & & & \\
\hline & & & -A_{k+1} & \ldots & -A_{n} \\
& & & & . & \\
& & & -A_{n} & &
\end{array}\right), \quad k=1, \ldots, n-1, \\
& P_{n+1}=\left(\begin{array}{cccc} 
& & & A_{0} \\
& & A_{0} & A_{1} \\
& . & \vdots & \vdots \\
A_{0} & \ldots & A_{n-2} & A_{n-1}
\end{array}\right) .
\end{aligned}
$$

For $i=1, \ldots, h$ and $j=1, \ldots,\left(m_{i}+1\right) n$, define functions $\psi_{i, j}$ on $\mathfrak{g}_{n}^{0}$ by

$$
\psi_{i, j}\left(x_{1}, \ldots, x_{n}\right):=\left.\varphi_{i, j}\right|_{\mathfrak{g}_{n}^{0}}=\left.\varphi_{i, j}\right|_{x_{0}=\mathrm{const}}
$$

(we do not define $\psi_{i, 0}$ because $\varphi_{i, 0}$ is constant on $\mathfrak{g}_{n}^{0}$ ). 


\section{Proposition 2.3.}

(i) Casimir functions of $P_{k+1}$ are $\psi_{i, j}, i=1, \ldots, h$, for $j=1,2, \ldots, k$ and for $j=m_{i} n+k+1$, $m_{i} n+k+2, \ldots,\left(m_{i}+1\right) n$.

(ii) Casimir functions of the combination $\lambda P_{k+1}-P_{k}$ are $\psi_{i, j}, i=1, \ldots, h$, for $j=1,2, \ldots, k-1$ and for $j=m_{i} n+k+1, m_{i} n+k+2, \ldots,\left(m_{i}+1\right) n$, and

$$
\lambda^{m_{i} n} \psi_{i, k}+\lambda^{m_{i} n-1} \psi_{i, k+1}+\cdots+\psi_{i, m_{i} n+k}, \quad i=1, \ldots, h .
$$

(iii) Let $F: \mathfrak{g}_{n}^{0} \rightarrow \mathbb{C}$ be a smooth function. The differential equation for the vector field $\left(\lambda P_{k+1}-\right.$ $\left.P_{k}\right) d F$ is expressed in Lax form as

$$
\frac{d}{d t} X_{\lambda}=\left[X_{\lambda}, \nabla_{k} F\right], \quad X_{\lambda}=\lambda^{n} X_{0}+\lambda^{n-1} X_{1}+\cdots+X_{n}
$$

(iv) Define the function $G_{i, k, j}$ to be

$$
G_{i, k, j}=-\left(\lambda^{j-1} \psi_{i, k}+\lambda^{j-2} \psi_{i, k+1}+\cdots+\psi_{i, k+j-1}\right) .
$$

Then, the equality

$$
P_{k+1} d \psi_{i, k+j}=P_{k} d \psi_{i, k+j-1}=\left(\lambda P_{k+1}-P_{k}\right) d G_{i, k, j}
$$

holds for $i=1, \ldots, h, j=1, \ldots, m_{i} n$ and $k=1, \ldots, n$. In particular, the vector field $P_{k+1} d \psi_{i, k+j}$ is independent of $k$ and the equation for it is expressed in Lax form as

$$
\frac{d}{d t} X_{\lambda}=\left[X_{\lambda}, \nabla_{k} G_{i, k, j}\right]
$$

(v) The vector fields $P_{k+1} d \psi_{i, k+j}$ for $i=1, \ldots, h$ and $j=1, \ldots, m_{i} n$ commute with each other (note that it is zero when $j \notin\left\{1, \ldots, m_{i} n\right\}$ ).

Proof. (i) and (ii) can be verified by a straightforward calculation with the aid of Proposition 2.1(v). To prove (iii), note that the vector field $P_{k+1} d F$ is written as

$$
P_{k+1} d F=\left(\begin{array}{ccc|ccc} 
& & {\left[X_{0}, \cdot\right]} & & & \\
& \cdot & \vdots & & & \\
{\left[X_{0}, \cdot\right]} & \ldots & {\left[X_{k-1}, \cdot\right]} & & & \\
\hline & & & -\left[X_{k+1}, \cdot\right] & \ldots & -\left[X_{n}, \cdot\right] \\
& & \vdots & . \cdot & \\
& & -\left[X_{n}, \cdot\right] & &
\end{array}\right)\left(\begin{array}{c}
\nabla_{1} F \\
\vdots \\
\nabla_{k} F \\
\nabla_{k+1} F \\
\vdots \\
\nabla_{n} F
\end{array}\right),
$$

and similarly for $P_{k} d F$. Using them, write down the equation of $X_{j}$ for the vector field $\left(\lambda P_{k+1}-\right.$ $\left.P_{k}\right) d F$. For example, the equation for $X_{1}$ is $d X_{1} / d t=\lambda\left[X_{0}, \nabla_{k} F\right]-\left[X_{0}, \nabla_{k-1} F\right]$. Summing up the equations of $\lambda^{n-j} X_{j}$ proves the desired result.

(iv) Since $\lambda^{m_{i} n} \psi_{i, k}+\cdots+\psi_{i, m_{i} n+k}$ is the Casimir of $\lambda P_{k+1}-P_{k}$, we have

$$
\left(\lambda P_{k+1}-P_{k}\right) d\left(\lambda^{m_{i} n} \psi_{i, k}+\lambda^{m_{i} n-1} \psi_{i, k+1}+\cdots+\psi_{i, m_{i} n+k}\right)=0 .
$$

Expanding this yields the first equality. The second equality is confirmed by a straightforward calculation.

(v) Due to Part (iv), we can assume that $k=n$. Because of the property $\left[P_{n+1} d F, P_{n+1} d G\right]=$ $P_{n+1} d\{G, F\}$ of a Poisson bracket (the left hand side is the Lie bracket for vector fields), it is 
sufficient to show the equality $\left\{\psi_{i^{\prime}, j^{\prime}}, \psi_{i, j}\right\}_{n+1}=0$ for $i, i^{\prime}=1, \ldots, h$ and $j, j^{\prime}=1, \ldots,\left(m_{i}+1\right) n$. When $j=1, \ldots, n$, it is trivial because $\psi_{i, j}$ is the Casimir of $P_{n+1}$. Next, we have

$$
\begin{aligned}
& \left\{\lambda^{m_{i} n} \psi_{i^{\prime}, k}+\lambda^{m_{i} n-1} \psi_{i^{\prime}, k+1}+\cdots+\psi_{i^{\prime}, m_{i} n+k}, \psi_{i, n+j}\right\}_{n+1} \\
& \quad=\left\langle d\left(\lambda^{m_{i} n} \psi_{i^{\prime}, k}+\lambda^{m_{i} n-1} \psi_{i^{\prime}, k+1}+\cdots+\psi_{i^{\prime}, m_{i} n+k}\right), P_{n+1} d \psi_{i, n+j}\right\rangle \\
& \quad=\left\langle d\left(\lambda^{m_{i} n} \psi_{i^{\prime}, k}+\lambda^{m_{i} n-1} \psi_{i^{\prime}, k+1}+\cdots+\psi_{i^{\prime}, m_{i} n+k}\right), P_{k+1} d \psi_{i, k+j}\right\rangle \\
& \quad=\left\langle d\left(\lambda^{m_{i} n} \psi_{i^{\prime}, k}+\lambda^{m_{i} n-1} \psi_{i^{\prime}, k+1}+\cdots+\psi_{i^{\prime}, m_{i} n+k}\right),\left(\lambda P_{k+1}-P_{k}\right) d G_{i, k, j}\right\rangle \\
& \quad=-\left\langle d G_{i, k, j},\left(\lambda P_{k+1}-P_{k}\right) d\left(\lambda^{m_{i} n} \psi_{i^{\prime}, k}+\lambda^{m_{i} n-1} \psi_{i^{\prime}, k+1}+\cdots+\psi_{i^{\prime}, m_{i} n+k}\right)\right\rangle=0 .
\end{aligned}
$$

This provides

$$
\left\{\psi_{i^{\prime}, k}, \psi_{i, n+j}\right\}_{n+1}=\cdots=\left\{\psi_{i^{\prime}, m_{i} n+k}, \psi_{i, n+j}\right\}_{n+1}=0,
$$

for any $k=1, \ldots, n$ and any $j=1, \ldots, m_{i} n$, which completes the proof.

Theorem 2.4. Suppose that the constant $x_{0}$ for the definition of $\mathfrak{g}_{n}^{0}$ is chosen so that the functions $\left\{\psi_{i, j}\right\}_{i, j}$ are functionally independent. Then, the vector field $P_{k+1} d \psi_{i, k+j}$, which is independent of $k$, is completely integrable in the Liouville sense for any $i$ and $j$.

Proof. Recall $\operatorname{dim}(\mathfrak{g})=d$ and $\operatorname{rank}(\mathfrak{g})=h$. Thus, $\operatorname{dim}\left(\mathfrak{g}_{n}^{0}\right)=n d$. Since $P_{k+1}$ has $n h$ Casimir functions, the dimension of a symplectic leaf $S$ of $P_{k+1}$ is $n(d-h)$. On the leaf $S$, the vector fields $\left\{P_{k+1} d \psi_{i, k+j}\right\}_{i, j}$ define $n(d-h)$-dimensional Hamiltonian systems, among which nonzero vector fields are for $i=1, \ldots, h$ and $j=1, \ldots, m_{i} n$. Further, these nonzero vector fields commute with each other and they are linearly independent due to the assumption. The number of the nonzero vector fields is

$$
\sum_{i=1}^{h} m_{i} n=\frac{1}{2}(\operatorname{dim}(\mathfrak{g})-\operatorname{rank}(\mathfrak{g})) n=\frac{1}{2} n(d-h)=\frac{1}{2} \operatorname{dim}(S) .
$$

Hence, the Liouville theorem shows that the vector fields are integrable.

In what follows, we suppose the above assumption; the constant $x_{0}$ for the definition of $\mathfrak{g}_{n}^{0}$ is chosen so that the functions $\left\{\psi_{i, j}\right\}_{i, j}$ are functionally independent. That is, the differentials $\left\{d \psi_{i, j}\right\}_{i, j}$ are linearly independent except for finite points.

\subsection{Symplectic reduction}

The next purpose is to perform a symplectic reduction $[9,14,15,16]$.

Theorem 2.5. The h-dimensional distribution $D$ defined by

$$
D=\operatorname{span}\left\{P_{k} d \psi_{i, k} \mid i=1, \ldots, h\right\}
$$

is integrable in the Frobenius sense. The vector fields $P_{k} d \psi_{i, k}$ are linear for $i=1, \ldots, h$.

Proof. The first statement follows from Proposition 2.3(v). Since $P_{k} d \psi_{i, k}$ is independent of $k$, we obtain $P_{k} d \psi_{i, k}=P_{1} d \psi_{i, 1}$. Since $\operatorname{wdeg}\left(\psi_{i, 1}\right)=1, d \psi_{1, i}$ is a constant, while $P_{1}$ is linear in $\left(x_{1}, \ldots, x_{n}\right)$.

The differential equation for $P_{k} d \psi_{i, k}=P_{1} d \psi_{i, 1}$ is given by

$$
\frac{d}{d t} X_{\lambda}=\left[X_{\lambda}, \nabla_{1} G_{i, 1,1}\right]=\left[\nabla_{1} \psi_{i, 1}, X_{\lambda}\right]
$$


Since $\nabla_{1} \psi_{i, 1}$ is independent of $\lambda$, this is decomposed as

$$
\frac{d}{d t} X_{k}=\left[\nabla_{1} \psi_{i, 1}, X_{k}\right], \quad k=1, \ldots, n
$$

In coordinates, it is expressed as

$$
\frac{d x_{k}}{d t}=-A_{k} \frac{\partial \psi_{i, 1}}{\partial x_{1}}\left(x_{1}\right), \quad k=1, \ldots, n .
$$

Let us consider the orbit space $\pi: \mathfrak{g}_{n}^{0} \rightarrow \mathfrak{g}_{n}^{0} / D$, which is a smooth manifold if points on $\mathfrak{g}_{n}^{0}$ at which $\operatorname{dim}(D)<h$ is removed if necessary. The Marsden-Ratiu reduction theorem [9] states that the orbit space $\mathfrak{g}_{n}^{0} / D$ is again a multi-Poisson manifold with compatible Poisson tensors denoted by $\widetilde{P}_{1}, \ldots, \widetilde{P}_{n+1}$. They are defined by $\widetilde{P}_{k}=\pi_{*} P_{k} \pi^{*}$. Let $\{,\}_{k}$ and $\{,\}_{k}^{\prime}$ be Poisson brackets associated with $P_{k}$ and $\widetilde{P}_{k}$, respectively. For a function $F$ on $\mathfrak{g}_{n}^{0}$ which is constant along each integral manifold of $D$, a function $\widetilde{F}$ on $\mathfrak{g}_{n}^{0} / D$ is well-defined through $\widetilde{F} \circ \pi=\pi^{*} \widetilde{F}=F$. Conversely, for a function $\widetilde{F}$ on $\mathfrak{g}_{n}^{0} / D$, we can find a function $F$ on $\mathfrak{g}_{n}^{0}$, which is constant along $D$, such that $\widetilde{F} \circ \pi=F$. Then, $\{,\}_{k}^{\prime}$ is given by $\{\widetilde{F}, \widetilde{G}\}_{k}^{\prime} \circ \pi=\{F, G\}_{k}$.

Because of Proposition 2.3(v), $\psi_{i, j}$ is constant along each integral manifold of $D$ and the projection $\widetilde{\psi}_{i, j}$ is well-defined. The projected vector field is given by $\widetilde{P}_{k} d \widetilde{\psi}_{i, j}=\pi_{*}\left(P_{k} d \psi_{i, j}\right)$.

It is convenient to realize $\mathfrak{g}_{n}^{0} / D$ as a submanifold of $\mathfrak{g}_{n}^{0}$. Let $\sigma: \mathfrak{g}_{n}^{0} / D \rightarrow \mathfrak{g}_{n}^{0}$ be a smooth section. The image $\sigma\left(\mathfrak{g}_{n}^{0} / D\right)$ is a submanifold of $\mathfrak{g}_{n}^{0}$ which is diffeomorphic to $\mathfrak{g}_{n}^{0} / D$. In Proposition 2.6(iii) below, $\mathfrak{g}_{n}^{0} / D$ is identified with a submanifold in this manner.

\section{Proposition 2.6.}

(i) Casimir functions of $\widetilde{P}_{k+1}$ are $\widetilde{\psi}_{i, j}, i=1, \ldots, h$, for $j=1,2, \ldots, k+1$ and for $j=$ $m_{i} n+k+1, m_{i} n+k+2, \ldots,\left(m_{i}+1\right) n$.

(ii) Casimir functions of the combination $\lambda \widetilde{P}_{k+1}-\widetilde{P}_{k}$ are $\widetilde{\psi}_{i, j}, i=1, \ldots, h$, for $j=1,2, \ldots, k$ and for $j=m_{i} n+k+1, m_{i} n+k+2, \ldots,\left(m_{i}+1\right) n$, and

$$
\lambda^{m_{i} n-1} \widetilde{\psi}_{i, k+1}+\lambda^{m_{i} n-2} \widetilde{\psi}_{i, k+2}+\cdots+\widetilde{\psi}_{i, m_{i} n+k}, \quad i=1, \ldots, h .
$$

(iii) For a smooth function $\widetilde{F}: \mathfrak{g}_{n}^{0} / D \rightarrow \mathbb{C}$, there exist scalar-valued functions $\beta_{1}, \ldots, \beta_{h}: \mathfrak{g}_{n}^{0} / D$ $\rightarrow \mathbb{C}$ such that the equation for the vector field $\left(\lambda \widetilde{P}_{k+1}-\widetilde{P}_{k}\right) d \widetilde{F}$ is expressed in Lax form as

$$
\frac{d}{d t} \widetilde{X}_{\lambda}=\left[\widetilde{X}_{\lambda}, \nabla_{k} \widetilde{F}\right]-\sum_{i=1}^{h} \beta_{i}\left[\widetilde{X}_{\lambda}, \nabla_{1} \widetilde{\psi}_{i, 1}\right]=\left[\widetilde{X}_{\lambda}, \nabla_{k} \widetilde{F}-\sum_{i=1}^{h} \beta_{i} \nabla_{1} \widetilde{\psi}_{i, 1}\right]
$$

where $\widetilde{X}_{\lambda}=\left.X_{\lambda}\right|_{\mathfrak{g}_{n}^{0} / D}$ and $\nabla_{k} \widetilde{F}=\left.\left(\nabla_{k} F\right)\right|_{\mathfrak{g}_{n}^{0} / D}$.

(iv) Define the function $\widetilde{G}_{i, k, j}$ to be

$$
\widetilde{G}_{i, k, j}=-\left(\lambda^{j-1} \widetilde{\psi}_{i, k}+\lambda^{j-2} \widetilde{\psi}_{i, k+1}+\cdots+\widetilde{\psi}_{i, k+j-1}\right) .
$$

Then, the equality

$$
\widetilde{P}_{k+1} d \widetilde{\psi}_{i, k+j}=\widetilde{P}_{k} d \widetilde{\psi}_{i, k+j-1}=\left(\lambda \widetilde{P}_{k+1}-\widetilde{P}_{k}\right) d \widetilde{G}_{i, k, j}
$$

holds for $i=1, \ldots, h, j=2, \ldots, m_{i} n$ and $k=1, \ldots, n$.

(v) The vector fields $\widetilde{P}_{k+1} d \widetilde{\psi}_{i, k+j}$ for $i=1, \ldots, h$ and $j=2, \ldots, m_{i} n$ commute with each other (note that it is zero when $j \notin\left\{2, \ldots, m_{i} n\right\}$ ). 
Proof. (i) $\widetilde{\psi}_{i, j}$ for $j=1, \ldots, k$ and $j=m_{i} n+k+1, \ldots,\left(m_{i}+1\right) n$ are Casimir of $\widetilde{P}_{k+1}$ because they are Casimir of $P_{k+1}$. For $\widetilde{\psi}_{i, k+1}$, we have

$$
\left\{\widetilde{F}, \widetilde{\psi}_{i, k+1}\right\}_{k+1}^{\prime}=\left\{F, \psi_{i, k+1}\right\}_{k+1}=\left\langle d F, P_{k+1} d \psi_{i, k+1}\right\rangle=\left(P_{k} d \psi_{i, k}\right)(F) .
$$

The right hand side becomes zero because $F$ is constant along $D$.

(ii) The first statement (on $\widetilde{\psi}_{i, j}$ ) is trivial because they are common Casimir of $\widetilde{P}_{k+1}$ and $\widetilde{P}_{k}$. The last function $\lambda^{m_{i} n-1} \widetilde{\psi}_{i, k+1}+\cdots$ is a projection of the function given in Proposition 2.3(ii).

The results of (iv) and (v) are projections of those of Proposition 2.3(iv) and (v).

To prove (iii), $\mathfrak{g}_{n}^{0} / D$ is identified with a submanifold of $\mathfrak{g}_{n}^{0}$ as above. Put $F=\pi^{*} \widetilde{F}$. We have to calculate the projection of the vector field $\left[X_{\lambda}, \nabla_{k} F\right]$ onto $\mathfrak{g}_{n}^{0} / D$ (see Proposition 2.3(iii)). At first, we restrict the domain to $\mathfrak{g}_{n}^{0} / D$ as

$$
\left.\left[X_{\lambda}, \nabla_{k} F\right]\right|_{\mathfrak{g}_{n}^{0} / D}=\left[\left.X_{\lambda}\right|_{\mathfrak{g}_{n}^{0} / D},\left.\left(\nabla_{k} F\right)\right|_{\mathfrak{g}_{n}^{0} / D}\right]=\left[\widetilde{X}_{\lambda}, \nabla_{k} \widetilde{F}\right] .
$$

Since this is not tangent to $T\left(\mathfrak{g}_{n}^{0} / D\right)$, we calculate the projection of it according to the decomposition $T \mathfrak{g}_{n}^{0}=T\left(\mathfrak{g}_{n}^{0} / D\right) \oplus D$. Then, (iii) follows from the fact that the distribution $D$ is spanned by the vector fields of the form $\left[X_{\lambda}, \nabla_{1} \psi_{i, 1}\right]$.

\subsection{Isospectral deformation to isomonodromic deformation}

Now we have $\left(m_{i} n-1\right) h$ distinct vector fields on $\mathfrak{g}_{n}^{0} / D$

$$
\begin{aligned}
& j=2: \quad \widetilde{P}_{1} d \widetilde{\psi}_{i, 2}=\cdots=\widetilde{P}_{k+1} d \widetilde{\psi}_{i, k+2}=\cdots=\widetilde{P}_{n+1} d \widetilde{\psi}_{i, n+2},
\end{aligned}
$$

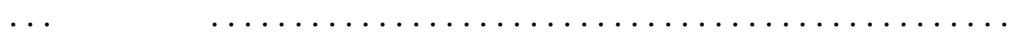

$$
\begin{aligned}
& j=j: \quad \widetilde{P}_{1} d \widetilde{\psi}_{i, j}=\cdots=\widetilde{P}_{k+1} d \widetilde{\psi}_{i, k+j}=\cdots=\widetilde{P}_{n+1} d \widetilde{\psi}_{i, n+j},
\end{aligned}
$$

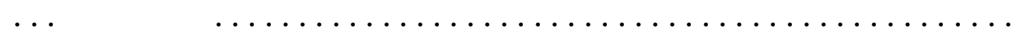

$$
\begin{aligned}
& j=m_{i} n: \quad \widetilde{P}_{1} d \widetilde{\psi}_{i, m_{i} n}=\cdots=\widetilde{P}_{k+1} d \widetilde{\psi}_{i, k+m_{i} n}=\cdots=\widetilde{P}_{n+1} d \widetilde{\psi}_{i,\left(m_{i}+1\right) n} .
\end{aligned}
$$

They are $(n d-h)$-dimensional integrable systems. For fixed $k$, a symplectic leaf of the Poisson structure $\widetilde{P}_{k}$ is given by a level surface of Casimir functions as

$$
S_{k}=\left\{\widetilde{\psi}_{i, j}=\alpha_{i, j} \text { (const) } \mid i=1, \ldots, h, j=1, \ldots, k, j=m_{i} n+k, \ldots,\left(m_{i}+1\right) n\right\} .
$$

Restricted on the symplectic leaf, the vector fields become $(n d-n h-2 h)$-dimensional completely integrable Hamiltonian systems of the form

$$
\begin{aligned}
& \widetilde{P}_{k+1} d \widetilde{\psi}_{i, k+j}: \frac{d}{d t} \widetilde{X}_{\lambda}=\left[\widetilde{X}_{\lambda}, A_{\lambda}\right] \quad \text { on } S_{k} \\
& A_{\lambda}:=\nabla_{k} \widetilde{G}_{i, k, j}-\sum_{i=1}^{h} \beta_{i} \nabla_{1} \widetilde{\psi}_{i, 1} .
\end{aligned}
$$

Both of $\widetilde{X}_{\lambda}$ and $A_{\lambda}$ depend on parameters $\left\{\alpha_{i, j}\right\}_{i, j}$ which define the symplectic leaf. Thus, we write $\widetilde{X}_{\lambda}$ as $\widetilde{X}_{\lambda}(t, \alpha)$, where $\alpha$ denotes the collection of parameters $\alpha_{i, j}$.

Now suppose that there exists a parameter $\alpha_{i^{\prime}, j^{\prime}}$ such that the following condition holds

$$
\frac{\partial \widetilde{X}_{\lambda}}{\partial \alpha_{i^{\prime}, j^{\prime}}}(t, \alpha)=\lambda^{l} \frac{\partial A_{\lambda}}{\partial \lambda}
$$

for some integer $l$. Equation (2.2) is put together with equation (2.3) to yield

$$
\frac{\partial \widetilde{X}_{\lambda}}{\partial t}(t, \alpha)+\frac{\partial \widetilde{X}_{\lambda}}{\partial \alpha_{i^{\prime}, j^{\prime}}}(t, \alpha)=\left[\widetilde{X}_{\lambda}, A_{\lambda}\right]+\lambda^{l} \frac{\partial A_{\lambda}}{\partial \lambda} .
$$


Define the Lax matrix $L_{\lambda}$ by

$$
L_{\lambda}:=\left.\frac{1}{\lambda^{l}} \tilde{X}_{\lambda}(t, \alpha)\right|_{\alpha_{i^{\prime}, j^{\prime}}=t},
$$

where the parameter $\alpha_{i^{\prime}, j^{\prime}}$ satisfying the condition (2.3) is replaced by $t$. Then, the above equation is rewritten as

$$
\frac{d L_{\lambda}}{d t}=\left[L_{\lambda}, A_{\lambda}\right]+\frac{\partial A_{\lambda}}{\partial \lambda},
$$

which is known as the isomonodromic deformation equation. It is known that a system written as the isomonodromic deformation equation enjoys the Painlevé property. The function $\widetilde{\psi}_{i, k+j}$ restricted on $S_{k}$ will be a Hamiltonian function of the Painlevé equation after replacing $\alpha_{i^{\prime}, j^{\prime}} \mapsto t$ and changing to Darboux's coordinates if necessary.

For ABCD-type simple Lie algebras, the dimensions of Painlevé systems obtained in this manner are summarized in Table 3. In particular, the dimension is 2 when $\mathfrak{g}=\mathfrak{s l}_{2}, n=2$, and it is 4 when $\mathfrak{g}=\mathfrak{s l}_{2}, n=3$ or $\mathfrak{g}=\mathfrak{s o}_{5}, n=1$. From the next sections, we will demonstrate our method for these cases. In particular, the Hamiltonian functions shown in Section 1 will be obtained.

Table 3. The dimensions of Painlevé systems.

\begin{tabular}{|c|c|c|c|c|c|}
\hline & & dimension & $h=1$ & $h=2$ & $h=3$ \\
\hline \hline $\mathrm{A}_{h}(h \geq 1)$ & $\mathfrak{s l}_{h+1}$ & $n h^{2}+n h-2 h$ & $2 n-2$ & $6 n-4$ & $12 n-6$ \\
\hline $\mathrm{B}_{h}(h \geq 2)$ & $\mathfrak{s o}_{2 h+1}$ & $2 n h^{2}-2 h$ & - & $8 n-4$ & $18 n-6$ \\
\hline $\mathrm{C}_{h}(h \geq 3)$ & $\mathfrak{s p}_{2 h}$ & $2 n h^{2}-2 h$ & - & - & $18 n-6$ \\
\hline $\mathrm{D}_{h}(h \geq 4)$ & $\mathfrak{s o}_{2 h}$ & $2 n h^{2}-2 n h-2 h$ & - & - & - \\
\hline
\end{tabular}

\section{2-dimensional Painlevé equations: $\mathfrak{g}=\mathfrak{s l}_{2}, n=2$}

In this case, a general element of $\mathfrak{g}_{n}$ is written by

$$
X_{\lambda}=\lambda^{2}\left(\begin{array}{cc}
u_{0} & v_{0} \\
w_{0} & -u_{0}
\end{array}\right)+\lambda\left(\begin{array}{cc}
u_{1} & v_{1} \\
w_{1} & -u_{1}
\end{array}\right)+\left(\begin{array}{cc}
u_{2} & v_{2} \\
w_{2} & -u_{2}
\end{array}\right) \text {. }
$$

The Painlevé equation obtained by our method depends on a choice of $x_{0}=\left(u_{0}, v_{0}, w_{0}\right)$. We consider the following two cases.

$$
\text { (I) }\left(\begin{array}{cc}
u_{0} & v_{0} \\
w_{0} & -u_{0}
\end{array}\right)=\left(\begin{array}{cc}
1 & 0 \\
0 & -1
\end{array}\right), \quad \text { (II) }\left(\begin{array}{cc}
u_{0} & v_{0} \\
w_{0} & -u_{0}
\end{array}\right)=\left(\begin{array}{ll}
0 & 0 \\
1 & 0
\end{array}\right) \text {. }
$$

From the former case, we will obtain the second and fourth Painlevé equations $\mathrm{P}_{\mathrm{II}}$, $\mathrm{P}_{\mathrm{IV}}$, and from the latter one, we will obtain the first and second Painlevé equations $\mathrm{P}_{\mathrm{I}}, \mathrm{P}_{\mathrm{II}}$.

\subsection{Case (I)}

In this case, the functions $\psi_{i, j}=\psi_{j}(\operatorname{since} h=\operatorname{rank}(\mathfrak{g})=1$, we omit the subscript $i)$ are given by

$$
\begin{array}{ll}
\psi_{1}=2 u_{1}, & \psi_{2}=2 u_{2}+u_{1}^{2}+v_{1} w_{1}, \\
\psi_{3}=2 u_{1} u_{2}+v_{2} w_{1}+v_{1} w_{2}, & \psi_{4}=u_{2}^{2}+v_{2} w_{2} .
\end{array}
$$


(see Example 2.2). The differential equation (2.1) defining the distribution $D$ is $u_{j}^{\prime}=0, v_{j}^{\prime}=2 v_{j}$, $w_{j}^{\prime}=-2 w_{j}$ for $j=1,2$. This is solved as a function of $w_{1}$ as

$$
u_{1}=U_{1}, \quad u_{2}=U_{2}, \quad v_{1}=V_{2} / w_{1}, \quad v_{2}=V_{3} / w_{1}, \quad w_{2}=W_{1} w_{1},
$$

where $U_{1}, U_{2}, V_{2}, V_{3}, W_{1}$ are integral constants (initial values at $w_{1}=1$ ), for which the subscripts are given so that they are consistent with the weighted degrees (for example, since wdeg $\left(v_{2} w_{1}\right)=$ $2+1=3$, the weighted degree of $V_{3}$ is three). This relation defines a coordinate transformation

$$
\left(u_{1}, v_{1}, w_{1}, u_{2}, v_{2}, w_{2}\right) \mapsto\left(U_{1}, V_{2}, w_{1}, U_{2}, V_{3}, W_{1}\right)
$$

In the new coordinates, integral manifolds of the distribution $D$ are straight lines along $w_{1^{-}}$ axis. In particular, the subset $\left\{w_{1}=1\right\} \subset \mathfrak{g}_{n}^{0}$ gives the realization of the orbit space $\mathfrak{g}_{n}^{0} / D$ as a submanifold and $\left(U_{1}, V_{2}, U_{2}, V_{3}, W_{1}\right)$ provides a global coordinate system of $\mathfrak{g}_{n}^{0} / D$.

At this stage, we have on $\mathfrak{g}_{n}^{0} / D$

$$
\begin{array}{ll}
\widetilde{\psi}_{1}=2 U_{1}, & \widetilde{\psi}_{2}=2 U_{2}+U_{1}^{2}+V_{2}, \\
\widetilde{\psi}_{3}=2 U_{1} U_{2}+V_{3}+V_{2} W_{1}, & \widetilde{\psi}_{4}=U_{2}^{2}+V_{3} W_{1},
\end{array}
$$

and three Poisson structures $\widetilde{P}_{1}$ (Casimirs are $\left.\widetilde{\psi}_{1}, \widetilde{\psi}_{3}, \widetilde{\psi}_{4}\right), \widetilde{P}_{2}\left(\right.$ Casimirs are $\left.\widetilde{\psi}_{1}, \widetilde{\psi}_{2}, \widetilde{\psi}_{4}\right)$, $\widetilde{P}_{3}$ (Casimirs are $\widetilde{\psi}_{1}, \widetilde{\psi}_{2}, \widetilde{\psi}_{3}$ ), and vector fields $\widetilde{P}_{3} d \widetilde{\psi}_{4}=\widetilde{P}_{2} d \widetilde{\psi}_{3}=\widetilde{P}_{1} d \widetilde{\psi}_{2}$ which are expressed as the Lax equation $d \widetilde{X}_{\lambda} / d t=\left[A_{\lambda}, \widetilde{X}_{\lambda}\right]$, where

$$
\begin{aligned}
& \widetilde{X}_{\lambda}=\lambda^{2}\left(\begin{array}{cc}
1 & 0 \\
0 & -1
\end{array}\right)+\lambda\left(\begin{array}{cc}
U_{1} & V_{2} \\
1 & -U_{1}
\end{array}\right)+\left(\begin{array}{cc}
U_{2} & V_{3} \\
W_{1} & -U_{2}
\end{array}\right), \\
& A_{\lambda}=\lambda\left(\begin{array}{cc}
1 & 0 \\
0 & -1
\end{array}\right)+\left(\begin{array}{cc}
U_{1} & V_{2} \\
1 & -U_{1}
\end{array}\right)-\left(\begin{array}{cc}
W_{1} & 0 \\
0 & -W_{1}
\end{array}\right) .
\end{aligned}
$$

The next purpose is to restrict the vector fields on a symplectic leaf. We will consider $\widetilde{P}_{3} d \widetilde{\psi}_{4}$ and $\widetilde{P}_{2} d \widetilde{\psi}_{3}$ separately $\left(\widetilde{P}_{1} d \widetilde{\psi}_{2}\right.$ will not be considered because there are no parameters satisfying (2.3)).

(i) Consider the vector field $\widetilde{P}_{3} d \widetilde{\psi}_{4}$. For the Poisson tensor $\widetilde{P}_{3}$, a symplectic leaf is defined by the level surface $\left\{\widetilde{\psi}_{j}=\right.$ const, $\left.j=1,2,3\right\}$. In order for the condition

$$
\frac{\partial \widetilde{X}_{\lambda}}{\partial \alpha}=\frac{\partial A_{\lambda}}{\partial \lambda}=\left(\begin{array}{cc}
1 & 0 \\
0 & -1
\end{array}\right)
$$

to be satisfied, we find that $U_{2}$ in $\widetilde{X}_{\lambda}$ has to include a parameter $\alpha$ which will be replaced by $t$ later. For this purpose, we take the symplectic leaf

$$
S=\left\{2 U_{1}=0,2 U_{2}+U_{1}^{2}+V_{2}=2 \alpha_{2}, 2 U_{1} U_{2}+V_{3}+V_{2} W_{1}=\alpha_{3}\right\} .
$$

Hence, we put $U_{1}=0, U_{2}=\alpha_{2}-V_{2} / 2, V_{3}=\alpha_{3}-V_{2} W_{1}$, and $\left(V_{2}, W_{1}\right)$ gives a global coordinate system for the symplectic leaf. Then, it turns out that $\widetilde{X}_{\lambda}$ satisfies the condition (3.1) with $\alpha=\alpha_{2}$ on the symplectic leaf. Finally, by replacing $\alpha_{2}$ by $t$, we obtain the isomonodromic deformation equation (2.4) with

$$
\begin{aligned}
& L_{\lambda}=\lambda^{2}\left(\begin{array}{cc}
1 & 0 \\
0 & -1
\end{array}\right)+\lambda\left(\begin{array}{cc}
0 & V_{2} \\
1 & 0
\end{array}\right)+\left(\begin{array}{cc}
t-V_{2} / 2 & \alpha_{3}-V_{2} W_{1} \\
W_{1} & -\left(t-V_{2} / 2\right)
\end{array}\right), \\
& A_{\lambda}=\lambda\left(\begin{array}{cc}
1 & 0 \\
0 & -1
\end{array}\right)+\left(\begin{array}{cc}
-W_{1} & V_{2} \\
1 & W_{1}
\end{array}\right) .
\end{aligned}
$$


The Poisson tensor $\widetilde{P}_{3}$ on the symplectic leaf with coordinates $\left(V_{2}, W_{1}\right)$ is given by

$$
\widetilde{P}_{3}=\left(\begin{array}{cc}
0 & 2 \\
-2 & 0
\end{array}\right)
$$

To change to Darboux's coordinates, we put $V_{2}=-2 p_{2}, W_{1}=q_{1}$. Then, $\widetilde{P}_{3}$ becomes the canonical symplectic matrix. In the coordinates $\left(q_{1}, p_{2}\right)$, the isomonodromic deformation equation is a Hamiltonian system. The Hamiltonian function $\widetilde{\psi}_{4}$ for the vector field $\widetilde{P}_{3} d \widetilde{\psi}_{4}$ is written as

$$
\begin{aligned}
\widetilde{\psi}_{4} & =U_{2}^{2}+V_{3} W_{1}=\left(t-V_{2} / 2\right)^{2}+\left(\alpha_{3}-V_{2} W_{1}\right) W_{1} \\
& =p_{2}^{2}+2 q_{1}^{2} p_{2}+2 t p_{2}+\alpha_{3} q_{1}+t^{2} .
\end{aligned}
$$

This is reduced to the Hamiltonian function (1.2) of the second Painlevé equation by a certain coordinate change and the isomonodromic deformation equation (2.4) is equivalent to the second Painlevé equation.

(ii) Consider the vector field $\widetilde{P}_{2} d \widetilde{\psi}_{3}$. For the Poisson tensor $\widetilde{P}_{2}$, a symplectic leaf is defined by the level surface $\left\{\widetilde{\psi}_{j}=\right.$ const, $\left.j=1,2,4\right\}$. In order for the condition

$$
\frac{\partial \widetilde{X}_{\lambda}}{\partial \alpha}=\lambda \frac{\partial A_{\lambda}}{\partial \lambda}=\lambda\left(\begin{array}{cc}
1 & 0 \\
0 & -1
\end{array}\right)
$$

to be satisfied, we find that $U_{1}$ in $\widetilde{X}_{\lambda}$ has to include a parameter $\alpha$ which will be replaced by $t$ later, and the other components of $\widetilde{X}_{\lambda}$ cannot include $\alpha$. For this purpose, we take the symplectic leaf

$$
S=\left\{2 U_{1}=2 \alpha_{1}, 2 U_{2}+U_{1}^{2}+V_{2}=\alpha_{2}+\alpha_{1}^{2}, U_{2}^{2}+V_{3} W_{1}=\alpha_{4}\right\} .
$$

This relation is rewritten as

$$
U_{1}=\alpha_{1}, \quad V_{2}=\alpha_{2}-2 U_{2}, \quad V_{3}=\left(\alpha_{4}-U_{2}^{2}\right) / W_{1} .
$$

By substituting them, $\widetilde{X}_{\lambda}$ satisfies the condition (3.2) with $\alpha=\alpha_{1}$. Finally, by replacing $\alpha_{1}$ by $t$, we obtain the isomonodromic deformation equation (2.4).

The Poisson tensor $\widetilde{P}_{2}$ on the symplectic leaf with coordinates $\left(U_{2}, W_{1}\right)$ is given by

$$
\widetilde{P}_{2}=\left(\begin{array}{cc}
0 & W_{1} \\
-W_{1} & 0
\end{array}\right)
$$

For Darboux's coordinates, we put $U_{2}=p_{1} q_{1}-\beta_{2}$ and $W_{1}=q_{1}$, where $\beta_{2}$ is an arbitrary constant. Then, $\widetilde{P}_{2}$ is transformed to the canonical symplectic matrix. In the coordinates $\left(q_{1}, p_{1}\right)$, the isomonodromic deformation equation is a Hamiltonian system. The Hamiltonian function $\widetilde{\psi}_{3}$ for the vector field $\widetilde{P}_{2} d \widetilde{\psi}_{3}$ is written as

$$
\begin{aligned}
\widetilde{\psi}_{3} & =2 U_{1} U_{2}+V_{3}+V_{2} W_{1}=2 t U_{2}+\left(\alpha_{4}-U_{2}^{2}\right) / W_{1}+\left(\alpha_{2}-2 U_{2}\right) W_{1} \\
& =-p_{1}^{2} q_{1}-2 p_{1} q_{1}^{2}+2 t p_{1} q_{1}+2 \beta_{2} p_{1}+\left(\alpha_{2}+2 \beta_{2}\right) q_{1}-2 \beta_{2} t+\frac{\alpha_{4}-\beta_{2}^{2}}{q_{1}} .
\end{aligned}
$$

We choose the free parameter $\beta_{2}$ to be $\alpha_{4}=\beta_{2}^{2}$ so that the Hamiltonian becomes a polynomial. This is the Hamiltonian function (1.3) of the fourth Painlevé equation up to some scaling. The isomonodromic deformation equation (2.4) is equivalent to the fourth Painlevé equation, where

$$
\begin{aligned}
& L_{\lambda}=\lambda\left(\begin{array}{cc}
1 & 0 \\
0 & -1
\end{array}\right)+\left(\begin{array}{cc}
t & \alpha_{2}+2 \beta_{2}-2 p_{1} q_{1} \\
1 & -t
\end{array}\right)+\frac{1}{\lambda}\left(\begin{array}{cc}
p_{1} q_{1}-\beta_{2} & -p_{1}^{2} q_{1}+2 \beta_{2} p_{1} \\
q_{1} & -\left(p_{1} q_{1}-\beta_{2}\right)
\end{array}\right), \\
& A_{\lambda}=\lambda\left(\begin{array}{cc}
1 & 0 \\
0 & -1
\end{array}\right)+\left(\begin{array}{cc}
t-q_{1} & \alpha_{2}+2 \beta_{2}-2 p_{1} q_{1} \\
1 & -\left(t-q_{1}\right)
\end{array}\right) .
\end{aligned}
$$




\subsection{Case (II)}

In this case, the functions $\psi_{i, j}=\psi_{j}$ are given by

$$
\begin{array}{ll}
\psi_{1}=v_{1}, & \psi_{2}=u_{1}^{2}+v_{2}+v_{1} w_{1}, \\
\psi_{3}=2 u_{1} u_{2}+v_{2} w_{1}+v_{1} w_{2}, & \psi_{4}=u_{2}^{2}+v_{2} w_{2} .
\end{array}
$$

The differential equation (2.1) defining the distribution $D$ is $u_{j}^{\prime}=-v_{j}, v_{j}^{\prime}=0, w_{j}^{\prime}=2 u_{j}$ for $j=1,2$. We can assume without loss of generality that $v_{1}=1$ by a suitable scaling of variables (indeed, $v_{1}$ is a common Casimir of $P_{1}, P_{2}, P_{3}$ ). Thus, the equations are solved as a function of $u_{1}$ as

$$
\begin{aligned}
& v_{1}=1, \quad v_{2}=V_{2}, \quad u_{2}=V_{2} u_{1}+U_{3}, \\
& w_{1}=-u_{1}^{2}+W_{2}, \quad w_{2}=-V_{2} u_{1}^{2}-2 U_{3} u_{1}+W_{4},
\end{aligned}
$$

where $V_{2}, U_{3}, W_{2}, W_{4}$ are integral constants (initial values at $u_{1}=0$ ). This relation defines a coordinate transformation

$$
\left(u_{1}, w_{1}, u_{2}, v_{2}, w_{2}\right) \mapsto\left(u_{1}, W_{2}, U_{3}, V_{2}, W_{4}\right) .
$$

In the new coordinates, integral manifolds of the distribution $D$ are straight lines along $u_{1^{-}}$ axis. In particular, the subset $\left\{u_{1}=0\right\} \subset \mathfrak{g}_{n}^{0}$ gives the realization of the orbit space $\mathfrak{g}_{n}^{0} / D$ and $\left(W_{2}, U_{3}, V_{2}, W_{4}\right)$ provides a global coordinate system of $\mathfrak{g}_{n}^{0} / D$ restricted to $v_{1}=1$.

On $\mathfrak{g}_{n}^{0} / D$, we have functions

$$
\widetilde{\psi}_{1}=1, \quad \widetilde{\psi}_{2}=V_{2}+W_{2}, \quad \widetilde{\psi}_{3}=V_{2} W_{2}+W_{4}, \quad \widetilde{\psi}_{4}=U_{3}^{2}+V_{2} W_{4},
$$

and three Poisson structures $\widetilde{P}_{1}$ (Casimirs are $\left.\widetilde{\psi}_{1}, \widetilde{\psi}_{3}, \widetilde{\psi}_{4}\right), \widetilde{P}_{2}$ (Casimirs are $\left.\widetilde{\psi}_{1}, \widetilde{\psi}_{2}, \widetilde{\psi}_{4}\right)$, $\widetilde{P}_{3}$ (Casimirs are $\widetilde{\psi}_{1}, \widetilde{\psi}_{2}, \widetilde{\psi}_{3}$ ), and vector fields $\widetilde{P}_{3} d \widetilde{\psi}_{4}=\widetilde{P}_{2} d \widetilde{\psi}_{3}=\widetilde{P}_{1} d \widetilde{\psi}_{2}$ which are expressed as the Lax equation $d \widetilde{X}_{\lambda} / d t=\left[A_{\lambda}, \widetilde{X}_{\lambda}\right]$, where

$$
\begin{aligned}
& \widetilde{X}_{\lambda}=\lambda^{2}\left(\begin{array}{ll}
0 & 0 \\
1 & 0
\end{array}\right)+\lambda\left(\begin{array}{cc}
0 & 1 \\
W_{2} & 0
\end{array}\right)+\left(\begin{array}{cc}
U_{3} & V_{2} \\
W_{4} & -U_{3}
\end{array}\right), \\
& A_{\lambda}=\lambda\left(\begin{array}{ll}
0 & 0 \\
1 & 0
\end{array}\right)+\left(\begin{array}{cc}
0 & 1 \\
W_{2} & 0
\end{array}\right)-\left(\begin{array}{cc}
0 & 0 \\
V_{2} & 0
\end{array}\right) .
\end{aligned}
$$

The next purpose is to restrict the vector fields on a symplectic leaf.

(i) Consider the vector field $\widetilde{P}_{3} d \widetilde{\psi}_{4}$. For the Poisson tensor $\widetilde{P}_{3}$, a symplectic leaf is defined by the level surface $\left\{\widetilde{\psi}_{j}=\right.$ const, $\left.j=1,2,3\right\}$. For the condition

$$
\frac{\partial \widetilde{X}_{\lambda}}{\partial \alpha}=\frac{\partial A_{\lambda}}{\partial \lambda}=\left(\begin{array}{ll}
0 & 0 \\
1 & 0
\end{array}\right)
$$

we find that $W_{4}$ in $\widetilde{X}_{\lambda}$ has to include a parameter $\alpha$. To this end, we take the symplectic leaf as

$$
S=\left\{\widetilde{\psi}_{1}=1, \widetilde{\psi}_{2}=V_{2}+W_{2}=0, \widetilde{\psi}_{3}=V_{2} W_{2}+W_{4}=\alpha_{4}\right\} .
$$

Hence, we put $V_{2}=-W_{2}, W_{4}=\alpha_{4}+W_{2}^{2}$, so that $\left(W_{2}, U_{3}\right)$ gives a global coordinate system on the leaf. Then, $\widetilde{X}_{\lambda}$ satisfies the condition (3.3) with $\alpha=\alpha_{4}$. Finally, by replacing $\alpha_{4}$ by $t$, we obtain the isomonodromic deformation equation (2.4) with

$$
\begin{aligned}
& L_{\lambda}=\lambda^{2}\left(\begin{array}{ll}
0 & 0 \\
1 & 0
\end{array}\right)+\lambda\left(\begin{array}{cc}
0 & 1 \\
W_{2} & 0
\end{array}\right)+\left(\begin{array}{cc}
U_{3} & -W_{2} \\
t+W_{2}^{2} & -U_{3}
\end{array}\right), \\
& A_{\lambda}=\lambda\left(\begin{array}{ll}
0 & 0 \\
1 & 0
\end{array}\right)+\left(\begin{array}{cc}
0 & 1 \\
2 W_{2} & 0
\end{array}\right) .
\end{aligned}
$$


The Poisson tensor $\widetilde{P}_{3}$ on the symplectic leaf is given by

$$
\widetilde{P}_{3}=\left(\begin{array}{cc}
0 & 1 \\
-1 & 0
\end{array}\right)
$$

which is already in canonical form. On the symplectic leaf, the function $\widetilde{\psi}_{4}$ is written as

$$
\widetilde{\psi}_{4}=U_{3}^{2}+V_{2} W_{4}=U_{3}^{2}-W_{2}^{3}-t W_{2}
$$

This is the Hamiltonian function (1.1) of the first Painlevé equation (up to some scaling) and the isomonodromic deformation equation (2.4) coincides with the first Painlevé equation.

(ii) For the vector field $\widetilde{P}_{2} d \widetilde{\psi}_{3}$, we again obtain the second Painlevé equation and the detailed calculation is omitted.

\section{4-dimensional Painlevé equations: $\mathfrak{g}=\mathfrak{s l}_{2}, n=3$}

In this case, a general element of $\mathfrak{g}_{n}$ is written as

$$
X_{\lambda}=\lambda^{3}\left(\begin{array}{cc}
u_{0} & v_{0} \\
w_{0} & -u_{0}
\end{array}\right)+\lambda^{2}\left(\begin{array}{cc}
u_{1} & v_{1} \\
w_{1} & -u_{1}
\end{array}\right)+\lambda\left(\begin{array}{cc}
u_{2} & v_{2} \\
w_{2} & -u_{2}
\end{array}\right)+\left(\begin{array}{cc}
u_{3} & v_{3} \\
w_{3} & -u_{3}
\end{array}\right) \text {. }
$$

For the definition of $\mathfrak{g}_{n}^{0}$, we again consider the following two cases.

$$
\text { (I) }\left(\begin{array}{cc}
u_{0} & v_{0} \\
w_{0} & -u_{0}
\end{array}\right)=\left(\begin{array}{cc}
1 & 0 \\
0 & -1
\end{array}\right), \quad \text { (II) }\left(\begin{array}{cc}
u_{0} & v_{0} \\
w_{0} & -u_{0}
\end{array}\right)=\left(\begin{array}{ll}
0 & 0 \\
1 & 0
\end{array}\right) \text {. }
$$

From the former case, we will obtain Hamiltonian functions (1.9), (1.10), (1.11), and from the latter one, we will obtain (1.7), (1.8), (1.12).

\subsection{Case (I)}

In this case, the functions $\psi_{i, j}=\psi_{j}($ since $h=\operatorname{rank}(\mathfrak{g})=1$, we omit the subscript $i$ ) are given by

$$
\begin{aligned}
& \psi_{1}=2 u_{1} \\
& \psi_{2}=2 u_{2}+u_{1}^{2}+v_{1} w_{1} \\
& \psi_{3}=2 u_{1} u_{2}+2 u_{3}+v_{2} w_{1}+v_{1} w_{2} \\
& \psi_{4}=u_{2}^{2}+2 u_{1} u_{3}+v_{3} w_{1}+v_{2} w_{2}+v_{1} w_{3} \\
& \psi_{5}=2 u_{2} u_{3}+v_{3} w_{2}+v_{2} w_{3} \\
& \psi_{6}=u_{3}^{2}+v_{3} w_{3}
\end{aligned}
$$

The differential equation (2.1) defining the distribution $D$ is $u_{j}^{\prime}=0, v_{j}^{\prime}=2 v_{j}, w_{j}^{\prime}=-2 w_{j}$ for $j=1,2,3$. This is solved as a function of $w_{1}$ as

$$
\begin{aligned}
& u_{1}=U_{1}, \quad u_{2}=U_{2}, \quad u_{3}=U_{3}, \\
& v_{1}=V_{2} / w_{1}, \quad v_{2}=V_{3} / w_{1}, \quad v_{3}=V_{4} / w_{1}, \quad w_{2}=W_{1} w_{1}, \quad w_{3}=W_{2} w_{1},
\end{aligned}
$$

where $U_{1}, U_{2}, U_{3}, V_{2}, V_{3}, V_{4}, W_{1}, W_{2}$ are integral constants (initial values at $w_{1}=1$ ). This relation defines a coordinate transformation

$$
\left(u_{1}, v_{1}, w_{1}, u_{2}, v_{2}, w_{2}, u_{3}, v_{3}, w_{3}\right) \mapsto\left(U_{1}, V_{2}, w_{1}, U_{2}, V_{3}, W_{1}, U_{3}, V_{4}, W_{2}\right)
$$


In the new coordinates, integral manifolds of the distribution $D$ are straight lines along $w_{1^{-}}$ axis. In particular, the subset $\left\{w_{1}=1\right\} \subset \mathfrak{g}_{n}^{0}$ gives the realization of the orbit space $\mathfrak{g}_{n}^{0} / D$ as a submanifold and $\left(U_{1}, V_{2}, U_{2}, V_{3}, W_{1}, U_{3}, V_{4}, W_{2}\right)$ provides a global coordinate system of $\mathfrak{g}_{n}^{0} / D$.

At this stage, we have on $\mathfrak{g}_{n}^{0} / D$

$$
\begin{aligned}
\widetilde{\psi}_{1} & =2 U_{1}, \\
\widetilde{\psi}_{2} & =2 U_{2}+U_{1}^{2}+V_{2}, \\
\widetilde{\psi}_{3} & =2 U_{1} U_{2}+2 U_{3}+V_{3}+V_{2} W_{1}, \\
\widetilde{\psi}_{4} & =2 U_{1} U_{3}+U_{2}^{2}+V_{4}+V_{2} W_{2}+V_{3} W_{1}, \\
\widetilde{\psi}_{5} & =2 U_{2} U_{3}+V_{4} W_{1}+V_{3} W_{2}, \\
\widetilde{\psi}_{6} & =U_{3}^{2}+V_{4} W_{2},
\end{aligned}
$$

and two vector fields

$$
\begin{aligned}
& \widetilde{P}_{2} d \widetilde{\psi}_{3}=\widetilde{P}_{3} d \widetilde{\psi}_{4}=\widetilde{P}_{4} d \widetilde{\psi}_{5}, \\
& \widetilde{P}_{2} d \widetilde{\psi}_{4}=\widetilde{P}_{3} d \widetilde{\psi}_{5}=\widetilde{P}_{4} d \widetilde{\psi}_{6} .
\end{aligned}
$$

The differential equations of these vector fields are expressed in Lax form as

$$
\frac{\partial \widetilde{X}_{\lambda}}{\partial t_{1}}=\left[A_{1}, \widetilde{X}_{\lambda}\right], \quad \frac{\partial \widetilde{X}_{\lambda}}{\partial t_{2}}=\left[A_{2}, \tilde{X}_{\lambda}\right]
$$

respectively, where

$$
\begin{aligned}
& \widetilde{X}_{\lambda}=\lambda^{3}\left(\begin{array}{cc}
1 & 0 \\
0 & -1
\end{array}\right)+\lambda^{2}\left(\begin{array}{cc}
U_{1} & V_{2} \\
1 & -U_{1}
\end{array}\right)+\lambda\left(\begin{array}{cc}
U_{2} & V_{3} \\
W_{1} & -U_{2}
\end{array}\right)+\left(\begin{array}{cc}
U_{3} & V_{4} \\
W_{2} & -U_{3}
\end{array}\right), \\
& A_{1}=\lambda\left(\begin{array}{cc}
1 & 0 \\
0 & -1
\end{array}\right)+\left(\begin{array}{cc}
U_{1} & V_{2} \\
1 & -U_{1}
\end{array}\right)-\left(\begin{array}{cc}
W_{1} & 0 \\
0 & -W_{1}
\end{array}\right), \\
& A_{2}=\lambda^{2}\left(\begin{array}{cc}
1 & 0 \\
0 & -1
\end{array}\right)+\lambda\left(\begin{array}{cc}
U_{1} & V_{2} \\
1 & -U_{1}
\end{array}\right)+\left(\begin{array}{cc}
U_{2} & V_{3} \\
W_{1} & -U_{2}
\end{array}\right)-\left(\begin{array}{cc}
W_{2} & 0 \\
0 & -W_{2}
\end{array}\right) .
\end{aligned}
$$

The next purpose is to restrict the vector fields on a symplectic leaf.

(i) Consider the pair of vector fields $\widetilde{P}_{4} d \widetilde{\psi}_{5}$ and $\widetilde{P}_{4} d \widetilde{\psi}_{6}$. For the Poisson tensor $\widetilde{P}_{4}$, a symplectic leaf is defined by the level surface $\left\{\widetilde{\psi}_{j}=\right.$ const, $\left.j=1,2,3,4\right\}$. In order for the two conditions

$$
\frac{\partial \widetilde{X}_{\lambda}}{\partial \alpha}=\frac{\partial A_{1}}{\partial \lambda}=\left(\begin{array}{cc}
1 & 0 \\
0 & -1
\end{array}\right), \quad \frac{\partial \widetilde{X}_{\lambda}}{\partial \alpha^{\prime}}=\frac{\partial A_{2}}{\partial \lambda}=2 \lambda\left(\begin{array}{cc}
1 & 0 \\
0 & -1
\end{array}\right)+\left(\begin{array}{cc}
U_{1} & V_{2} \\
1 & -U_{1}
\end{array}\right)
$$

to be satisfied, we find that $U_{3}$ in $\widetilde{X}_{\lambda}$ has to include a parameter $\alpha$, which will be replaced by $t_{1}$ later, and $U_{2}$ and $W_{2}$ have to include a parameter $\alpha^{\prime}$, which will be replaced by $t_{2}$ later. For this purpose, we take the symplectic leaf

$$
S=\left\{\widetilde{\psi}_{1}=0, \widetilde{\psi}_{2}=4 \alpha_{2}, \widetilde{\psi}_{3}=2 \alpha_{3}, \widetilde{\psi}_{4}=\alpha_{4}+4 \alpha_{2}^{2}\right\}
$$

Further, we change the coordinate as $W_{2}=\widetilde{W}_{2}+\alpha_{2}$ because $W_{2}$ should include a parameter. Then, the above relation for $S$ is rearranged as

$$
\begin{aligned}
& U_{1}=0, \quad U_{2}=2 \alpha_{2}-\frac{1}{2} V_{2}, \quad U_{3}=\alpha_{3}-\frac{1}{2} V_{3}-\frac{1}{2} V_{2} W_{1}, \\
& V_{4}=\alpha_{4}+\alpha_{2} V_{2}-\frac{1}{4} V_{2}^{2}-V_{2} \widetilde{W}_{2}-V_{3} W_{1} .
\end{aligned}
$$


Substituting them into $\widetilde{X}_{\lambda}, A_{1}$ and $A_{2}$, we can verify the condition (4.4) with $\alpha=\alpha_{3}$ and $\alpha^{\prime}=\alpha_{2}$. By replacing $\alpha_{3}, \alpha_{2}$ by $t_{1}, t_{2}$, respectively, we obtain the isomonodromic deformation equations

$$
\frac{\partial L_{\lambda}}{\partial t_{1}}=\left[A_{1}, L_{\lambda}\right]+\frac{\partial A_{1}}{\partial \lambda}, \quad \frac{\partial L_{\lambda}}{\partial t_{2}}=\left[A_{2}, L_{\lambda}\right]+\frac{\partial A_{2}}{\partial \lambda}
$$

which are equations of $\left(V_{2}, V_{3}, W_{1}, \widetilde{W}_{2}\right)$ with two independent variables $t_{1}$, $t_{2}$, where

$$
\begin{aligned}
L_{\lambda}= & \lambda^{3}\left(\begin{array}{cc}
1 & 0 \\
0 & -1
\end{array}\right)+\lambda^{2}\left(\begin{array}{cc}
0 & V_{2} \\
1 & 0
\end{array}\right)+\lambda\left(\begin{array}{cc}
2 t_{2}-\frac{1}{2} V_{2} & V_{3} \\
W_{1} & -\left(2 t_{2}-\frac{1}{2} V_{2}\right)
\end{array}\right) \\
& +\left(\begin{array}{cc}
t_{1}-\frac{1}{2} V_{3}-\frac{1}{2} V_{2} W_{1} & \alpha_{4}+t_{2} V_{2}-\frac{1}{4} V_{2}^{2}-V_{2} \widetilde{W}_{2}-V_{3} W_{1} \\
\widetilde{W}_{2}+t_{2} & -\left(t_{1}-\frac{1}{2} V_{3}-\frac{1}{2} V_{2} W_{1}\right)
\end{array}\right), \\
A_{1}= & \lambda\left(\begin{array}{cc}
1 & 0 \\
0 & -1
\end{array}\right)+\left(\begin{array}{cc}
-W_{1} & V_{2} \\
1 & W_{1}
\end{array}\right), \\
A_{2}= & \lambda^{2}\left(\begin{array}{cc}
1 & 0 \\
0 & -1
\end{array}\right)+\lambda\left(\begin{array}{cc}
0 & V_{2} \\
1 & 0
\end{array}\right)+\left(\begin{array}{cc}
t_{2}-\frac{1}{2} V_{2}-\widetilde{W}_{2} & V_{3} \\
W_{1} & -\left(t_{2}-\frac{1}{2} V_{2}-\widetilde{W}_{2}\right)
\end{array}\right) .
\end{aligned}
$$

The Poisson tensor $\widetilde{P}_{4}$ on the symplectic leaf written in the coordinates $\left(V_{2}, V_{3}, W_{1}, \widetilde{W}_{2}\right)$ is given by

$$
\widetilde{P}_{4}=\left(\begin{array}{cccc}
0 & 0 & 0 & 2 \\
0 & 0 & 2 & 0 \\
0 & -2 & 0 & 0 \\
-2 & 0 & 0 & 0
\end{array}\right)
$$

The above two isomonodromic deformation equations can be written as Hamiltonian systems. The Hamiltonian functions of these equations are obtained by deleting $U_{1}, U_{2}, U_{3}, V_{4}$ from $\widetilde{\psi}_{5}$ and $\widetilde{\psi}_{6}$ by using the above relations, and changing to Darboux's coordinates by a scaling so that the above $\widetilde{P}_{4}$ is transformed to the canonical symplectic matrix. In this manner, we obtain Hamiltonian functions (1.9) of $\left(\mathrm{P}_{\mathrm{II}-2}\right)_{2}$ given in Section 1.

(ii) Consider the pair of vector fields $\widetilde{P}_{3} d \widetilde{\psi}_{4}$ and $\widetilde{P}_{3} d \widetilde{\psi}_{5}$. For the Poisson tensor $\widetilde{P}_{3}$, a symplectic leaf is defined by the level surface $\left\{\widetilde{\psi}_{j}=\right.$ const, $\left.j=1,2,3,6\right\}$. For the two conditions

$$
\frac{\partial \widetilde{X}_{\lambda}}{\partial \alpha}=\lambda \frac{\partial A_{1}}{\partial \lambda}=\lambda\left(\begin{array}{cc}
1 & 0 \\
0 & -1
\end{array}\right), \quad \frac{\partial \widetilde{X}_{\lambda}}{\partial \alpha^{\prime}}=\lambda \frac{\partial A_{2}}{\partial \lambda}=2 \lambda^{2}\left(\begin{array}{cc}
1 & 0 \\
0 & -1
\end{array}\right)+\lambda\left(\begin{array}{cc}
U_{1} & V_{2} \\
1 & -U_{1}
\end{array}\right),
$$

we find that $U_{2}$ in $\widetilde{X}_{\lambda}$ has to include a parameter $\alpha$, and $U_{1}$ and $W_{1}$ have to include a parameter $\alpha^{\prime}$. For this purpose, we take the symplectic leaf

$$
S=\left\{\widetilde{\psi}_{1}=4 \alpha_{1}, \widetilde{\psi}_{2}=2 \alpha_{2}+6 \alpha_{1}^{2}, \widetilde{\psi}_{3}=\alpha_{3}+4 \alpha_{1}\left(\alpha_{2}+\alpha_{1}^{2}\right), \widetilde{\psi}_{6}=\alpha_{6}\right\}
$$

Further, we change the coordinate as $W_{1}=\widetilde{W}_{1}+\alpha_{1}$. Then, the above relations for $S$ yield

$$
\begin{aligned}
& U_{1}=2 \alpha_{1}, \quad U_{2}=\alpha_{2}+\alpha_{1}^{2}-\frac{1}{2} V_{2}, \quad V_{3}=\alpha_{3}-2 U_{3}-V_{2} \widetilde{W}_{1}+\alpha_{1} V_{2}, \\
& V_{4}=\left(\alpha_{6}-U_{3}^{2}\right) / W_{2} .
\end{aligned}
$$

Substituting them into $\widetilde{X}_{\lambda}, A_{1}$ and $A_{2}$, we can verify the condition (4.6) with $\alpha=\alpha_{2}$ and $\alpha^{\prime}=\alpha_{1}$. By replacing $\alpha_{2}, \alpha_{1}$ by $t_{1}, t_{2}$, respectively, we obtain the isomonodromic deformation equations (4.5), which are equations of $\left(V_{2}, \widetilde{W}_{1}, U_{3}, W_{2}\right)$ with two independent variables $t_{1}, t_{2}$. 
The Poisson tensor $\widetilde{P}_{3}$ on the symplectic leaf expressed in the coordinates $\left(V_{2}, \widetilde{W}_{1}, U_{3}, W_{2}\right)$ is given by

$$
\widetilde{P}_{3}=\left(\begin{array}{cccc}
0 & 2 & 0 & 0 \\
-2 & 0 & 0 & 0 \\
0 & 0 & 0 & W_{2} \\
0 & 0 & -W_{2} & 0
\end{array}\right) .
$$

To change to Darboux's coordinates, put

$$
\left(V_{2}, \widetilde{W}_{1}, U_{3}, W_{2}\right)=\left(2 p_{1}, q_{1}, q_{2} p_{2}-\beta_{3}, p_{2}\right),
$$

where $\beta_{3}$ is an arbitrary parameter. In the new coordinates, we obtain

$$
\widetilde{P}_{3}=\left(\begin{array}{cccc}
0 & 1 & 0 & 0 \\
-1 & 0 & 0 & 0 \\
0 & 0 & 0 & 1 \\
0 & 0 & -1 & 0
\end{array}\right)
$$

Therefore, the two isomonodromic deformation equations (4.5) are Hamiltonian systems in this coordinate system. The Hamiltonian functions are obtained by deleting $\left(U_{1}, U_{2}, V_{3}, V_{4}\right)$ from $\widetilde{\psi}_{4}$ and $\widetilde{\psi}_{5}$ and by changing to the coordinates $\left(p_{1}, q_{1}, p_{2}, q_{2}\right)$. It is easy to verify that if we set $\beta_{3}^{2}=\alpha_{6}$, then two functions become polynomials, which give Hamiltonian functions (1.10) of $\left(\mathrm{P}_{\mathrm{IV}}\right)_{2}$ given in Section 1.

(iii) Consider the pair of vector fields $\widetilde{P}_{2} d \widetilde{\psi}_{3}$ and $\widetilde{P}_{2} d \widetilde{\psi}_{4}$. For the Poisson tensor $\widetilde{P}_{2}$, a symplectic leaf is defined by the level surface $\left\{\widetilde{\psi}_{j}=\right.$ const, $\left.j=1,2,5,6\right\}$. In this case, we cannot find an integer $l$ and a parameter $\alpha^{\prime}$ such that the condition

$$
\frac{\partial \widetilde{X}_{\lambda}}{\partial \alpha^{\prime}}=\lambda^{l} \frac{\partial A_{2}}{\partial \lambda}
$$

holds. Hence, we impose only one condition

$$
\frac{\partial \widetilde{X}_{\lambda}}{\partial \alpha}=\lambda^{2} \frac{\partial A_{1}}{\partial \lambda}=\lambda^{2}\left(\begin{array}{cc}
1 & 0 \\
0 & -1
\end{array}\right) \text {. }
$$

For it, $U_{1}$ in $\widetilde{X}_{\lambda}$ has to include a parameter $\alpha$. To this end, take the symplectic leaf

$$
S=\left\{\widetilde{\psi}_{1}=2 \alpha_{1}, \widetilde{\psi}_{2}=2 \alpha_{2}+\alpha_{1}^{2}, \widetilde{\psi}_{5}=\alpha_{5}, \widetilde{\psi}_{6}=\alpha_{6}\right\} .
$$

This is rearranged as

$$
\begin{aligned}
& U_{1}=\alpha_{1}, \quad V_{2}=2 \alpha_{2}-2 U_{2}, \quad V_{3}=\left(\alpha_{5}-2 U_{2} U_{3}-V_{4} W_{1}\right) / W_{2}, \\
& V_{4}=\left(\alpha_{6}-U_{3}^{2}\right) / W_{2} .
\end{aligned}
$$

Substituting them into $\widetilde{X}_{\lambda}$ and $A_{1}$, we can verify the condition (4.8) with $\alpha=\alpha_{1}$. By replacing $\alpha_{1}$ by $t$, we obtain the isomonodromic deformation equation

$$
\frac{\partial L_{\lambda}}{\partial t}=\left[A_{1}, L_{\lambda}\right]+\frac{\partial A_{1}}{\partial \lambda} .
$$

The Poisson tensor $\widetilde{P}_{2}$ on the symplectic leaf with coordinates $\left(U_{2}, W_{1}, U_{3}, W_{2}\right)$ is given by

$$
\widetilde{P}_{2}=\left(\begin{array}{cccc}
0 & W_{1} & 0 & W_{2} \\
-W_{1} & 0 & -W_{2} & 0 \\
0 & W_{2} & 0 & 0 \\
-W_{2} & 0 & 0 & 0
\end{array}\right)
$$


To change to Darboux's coordinates, put

$$
\left(U_{2}, W_{1}, U_{3}, W_{2}\right)=\left(p_{1} q_{1}+p_{2} q_{2}-\beta_{2}, q_{1}, p_{1} q_{2}-\beta_{3}, q_{2}\right)
$$

where $\beta_{2}$ and $\beta_{3}$ are arbitrary parameters. In the new coordinates $\left(q_{1}, p_{1}, q_{2}, p_{2}\right), \widetilde{P}_{2}$ is reduced to the same form as (4.7). The isomonodromic deformation equation is a Hamiltonian system whose Hamiltonian function is $\widetilde{\psi}_{3}$ written in this coordinate system. It is easy to verify that if we set $\alpha_{6}=\beta_{3}^{2}$ and $\alpha_{5}=2 \beta_{2} \beta_{3}$, then $\widetilde{\psi}_{3}$ written in the coordinates $\left(q_{1}, p_{1}, q_{2}, p_{2}\right)$ becomes a polynomial. In this manner, the Hamiltonian function (1.11) given in Section 1 is obtained.

\subsection{Case (II)}

In this case, the functions $\psi_{i, j}=\psi_{j}$ are given by

$$
\begin{aligned}
& \psi_{1}=v_{1} \\
& \psi_{2}=u_{1}^{2}+v_{2}+v_{1} w_{1} \\
& \psi_{3}=2 u_{1} u_{2}+v_{3}+v_{2} w_{1}+v_{1} w_{2}, \\
& \psi_{4}=u_{2}^{2}+2 u_{1} u_{3}+v_{3} w_{1}+v_{2} w_{2}+v_{1} w_{3}, \\
& \psi_{5}=2 u_{2} u_{3}+v_{3} w_{2}+v_{2} w_{3} \\
& \psi_{6}=u_{3}^{2}+v_{3} w_{3}
\end{aligned}
$$

The differential equation (2.1) defining the distribution $D$ is $u_{j}^{\prime}=-v_{j}, v_{j}^{\prime}=0, w_{j}^{\prime}=2 u_{j}$ for $j=1,2,3$. We can assume without loss of generality that $v_{1}=1$ by a suitable scaling of variables. These equations are solved with respect to $u_{1}$ as

$$
\begin{array}{lrl}
v_{1}=1, \quad w_{1}=-u_{1}^{2}+W_{2}, & \\
u_{2}=V_{2} u_{1}+U_{3}, & v_{2}=V_{2}, & w_{2}=-V_{2} u_{1}^{2}-2 U_{3} u_{1}+W_{4}, \\
u_{3}=V_{4} u_{1}+U_{5}, & v_{3}=V_{4}, & w_{3}=-V_{4} u_{1}^{2}-2 U_{5} u_{1}+W_{6},
\end{array}
$$

where $W_{2}, U_{3}, V_{2}, W_{4}, U_{5}, V_{4}, W_{6}$ are integral constants (initial values at $u_{1}=0$ ). This relation defines a coordinate transformation

$$
\left(u_{1}, w_{1}, u_{2}, v_{2}, w_{2}, u_{3}, v_{3}, w_{3}\right) \mapsto\left(u_{1}, W_{2}, U_{3}, V_{2}, W_{4}, U_{5}, V_{4}, W_{6}\right) .
$$

In the new coordinates, integral manifolds of the distribution $D$ are straight lines along $u_{1^{-}}$ axis. In particular, the subset $\left\{u_{1}=0\right\} \subset \mathfrak{g}_{n}^{0}$ gives the realization of the orbit space $\mathfrak{g}_{n}^{0} / D$ as a submanifold and $\left(W_{2}, U_{3}, V_{2}, W_{4}, U_{5}, V_{4}, W_{6}\right)$ provides a global coordinate system of $\mathfrak{g}_{n}^{0} / D$ restricted to $v_{1}=1$.

On $\mathfrak{g}_{n}^{0} / D$, we have functions

$$
\begin{aligned}
\widetilde{\psi}_{1} & =1, \\
\widetilde{\psi}_{2} & =V_{2}+W_{2}, \\
\widetilde{\psi}_{3} & =V_{4}+W_{4}+V_{2} W_{2}, \\
\widetilde{\psi}_{4} & =U_{3}^{2}+W_{6}+V_{2} W_{4}+V_{4} W_{2}, \\
\widetilde{\psi}_{5} & =2 U_{3} U_{5}+V_{2} W_{6}+V_{4} W_{4}, \\
\widetilde{\psi}_{6} & =U_{5}^{2}+V_{4} W_{6},
\end{aligned}
$$

and two vector fields (4.1), (4.2) expressed in Lax form (4.3) with

$$
\widetilde{X}_{\lambda}=\lambda^{3}\left(\begin{array}{ll}
0 & 0 \\
1 & 0
\end{array}\right)+\lambda^{2}\left(\begin{array}{cc}
0 & 1 \\
W_{2} & 0
\end{array}\right)+\lambda\left(\begin{array}{cc}
U_{3} & V_{2} \\
W_{4} & -U_{3}
\end{array}\right)+\left(\begin{array}{cc}
U_{5} & V_{4} \\
W_{6} & -U_{5}
\end{array}\right),
$$




$$
\begin{aligned}
& A_{1}=\lambda\left(\begin{array}{ll}
0 & 0 \\
1 & 0
\end{array}\right)+\left(\begin{array}{cc}
0 & 1 \\
W_{2} & 0
\end{array}\right)-\left(\begin{array}{cc}
0 & 0 \\
V_{2} & 0
\end{array}\right) \\
& A_{2}=\lambda^{2}\left(\begin{array}{ll}
0 & 0 \\
1 & 0
\end{array}\right)+\lambda\left(\begin{array}{cc}
0 & 1 \\
W_{2} & 0
\end{array}\right)+\left(\begin{array}{cc}
U_{3} & V_{2} \\
W_{4} & -U_{3}
\end{array}\right)-\left(\begin{array}{cc}
0 & 0 \\
V_{4} & 0
\end{array}\right) .
\end{aligned}
$$

The next purpose is to restrict the vector fields on a symplectic leaf.

(i) Consider the pair of vector fields $\widetilde{P}_{4} d \widetilde{\psi}_{5}$ and $\widetilde{P}_{4} d \widetilde{\psi}_{6}$. For the Poisson tensor $\widetilde{P}_{4}$, a symplectic leaf is defined by the level surface $\left\{\widetilde{\psi}_{j}=\right.$ const, $\left.j=1,2,3,4\right\}$. In order for the two conditions

$$
\frac{\partial \widetilde{X}_{\lambda}}{\partial \alpha}=\frac{\partial A_{1}}{\partial \lambda}=\left(\begin{array}{cc}
0 & 0 \\
1 & 0
\end{array}\right), \quad \frac{\partial \widetilde{X}_{\lambda}}{\partial \alpha^{\prime}}=\frac{\partial A_{2}}{\partial \lambda}=2 \lambda\left(\begin{array}{cc}
0 & 0 \\
1 & 0
\end{array}\right)+\left(\begin{array}{cc}
0 & 1 \\
W_{2} & 0
\end{array}\right)
$$

to be satisfied, we find that $W_{6}$ in $\widetilde{X}_{\lambda}$ has to include a parameter $\alpha$, which will be replaced by $t_{1}$ later, and $W_{4}$ and $V_{4}$ have to include a parameter $\alpha^{\prime}$, which will be replaced by $t_{2}$ later. For this purpose, we take the symplectic leaf

$$
S=\left\{\widetilde{\psi}_{1}=1, \widetilde{\psi}_{2}=0, \widetilde{\psi}_{3}=3 \alpha_{4}, \widetilde{\psi}_{4}=\alpha_{6}\right\}
$$

Further, we change the coordinate as $W_{4}=\widetilde{W}_{4}+2 \alpha_{4}$. Then, the above relation for $S$ is rearranged as

$$
V_{2}=-W_{2}, \quad V_{4}=\alpha_{4}-\widetilde{W}_{4}+W_{2}^{2}, \quad W_{6}=\alpha_{6}-U_{3}^{2}+2 W_{2} \widetilde{W}_{4}-W_{2}^{3}+\alpha_{4} W_{2} .
$$

Substituting them into $\widetilde{X}_{\lambda}, A_{1}$ and $A_{2}$, we can verify the condition (4.9) with $\alpha=\alpha_{6}$ and $\alpha^{\prime}=\alpha_{4}$. By replacing $\alpha_{6}, \alpha_{4}$ by $t_{1}, t_{2}$, respectively, we obtain the isomonodromic deformation equations (4.5), which are equations of $\left(W_{2}, U_{3}, \widetilde{W}_{4}, U_{5}\right)$ with two independent variables $t_{1}, t_{2}$. The Poisson tensor $\widetilde{P}_{4}$ on the symplectic leaf written in this coordinate system is given by

$$
\widetilde{P}_{4}=\left(\begin{array}{cccc}
0 & 0 & 0 & 1 \\
0 & 0 & -1 & 0 \\
0 & 1 & 0 & W_{2} \\
-1 & 0 & -W_{2} & 0
\end{array}\right)
$$

To change to Darboux's coordinates, put

$$
\left(W_{2}, U_{3}, \widetilde{W}_{4}, U_{5}\right)=\left(q_{1}, p_{2}, q_{2}, p_{1}+p_{2} q_{1}\right) .
$$

Then, $\widetilde{P}_{4}$ is transformed to the canonical symplectic matrix. In the coordinates $\left(q_{1}, p_{1}, q_{2}, p_{2}\right)$, the above two isomonodromic deformation equations are Hamiltonian systems. The Hamiltonian functions are obtained by deleting $V_{2}, V_{4}, W_{6}$ from $\widetilde{\psi}_{5}$ and $\widetilde{\psi}_{6}$ by using the above relations, and changing to Darboux's coordinates. In this manner, we obtain Hamiltonian functions (1.7) of $\left(\mathrm{P}_{\mathrm{I}}\right)_{2}$ given in Section 1.

(ii) Consider the pair of vector fields $\widetilde{P}_{3} d \widetilde{\psi}_{4}$ and $\widetilde{P}_{3} d \widetilde{\psi}_{5}$. For the Poisson tensor $\widetilde{P}_{3}$, a symplectic leaf is defined by the level surface $\left\{\widetilde{\psi}_{j}=\right.$ const, $\left.j=1,2,3,6\right\}$. For the two conditions

$$
\frac{\partial \widetilde{X}_{\lambda}}{\partial \alpha}=\lambda \frac{\partial A_{1}}{\partial \lambda}=\lambda\left(\begin{array}{cc}
0 & 0 \\
1 & 0
\end{array}\right), \quad \frac{\partial \widetilde{X}_{\lambda}}{\partial \alpha^{\prime}}=\lambda \frac{\partial A_{2}}{\partial \lambda}=2 \lambda^{2}\left(\begin{array}{cc}
0 & 0 \\
1 & 0
\end{array}\right)+\lambda\left(\begin{array}{cc}
0 & 1 \\
W_{2} & 0
\end{array}\right),
$$

we find that $W_{4}$ in $\widetilde{X}_{\lambda}$ has to include a parameter $\alpha$, and $W_{2}$ and $V_{2}$ have to include a parameter $\alpha^{\prime}$. For this purpose, we take the symplectic leaf

$$
S=\left\{\widetilde{\psi}_{1}=1, \widetilde{\psi}_{2}=3 \alpha_{2}, \widetilde{\psi}_{3}=\alpha_{4}+3 \alpha_{2}^{2}, \widetilde{\psi}_{6}=\alpha_{10}\right\}
$$


Further, we change the coordinate as $V_{2}=\widetilde{V}_{2}+\alpha_{2}$. Then, the above relation for $S$ is rearranged as

$$
W_{2}=2 \alpha_{2}-\widetilde{V}_{2}, \quad W_{4}=\alpha_{4}+\alpha_{2}^{2}-\alpha_{2} \widetilde{V}_{2}-V_{4}+V_{2}^{2}, \quad W_{6}=\left(\alpha_{10}-U_{5}^{2}\right) / V_{4} .
$$

Substituting them into $\widetilde{X}_{\lambda}, A_{1}$ and $A_{2}$, we can verify the condition (4.10) with $\alpha=\alpha_{4}$ and $\alpha^{\prime}=\alpha_{2}$. By replacing $\alpha_{4}, \alpha_{2}$ by $t_{1}, t_{2}$, respectively, we obtain the isomonodromic deformation equations (4.5). The Poisson tensor $\widetilde{P}_{3}$ on the symplectic leaf written in the coordinates $\left(\widetilde{V}_{2}, U_{3}, V_{4}, U_{5}\right)$ is given by

$$
\widetilde{P}_{3}=\left(\begin{array}{cccc}
0 & 1 & 0 & 0 \\
-1 & 0 & 0 & 0 \\
0 & 0 & 0 & -V_{4} \\
0 & 0 & V_{4} & 0
\end{array}\right)
$$

To change to Darboux's coordinates, put

$$
\left(\widetilde{V}_{2}, U_{3}, V_{4}, U_{5}\right)=\left(p_{2}, q_{2}, p_{1}, q_{1} p_{1}-\alpha_{5}\right)
$$

where $\alpha_{5}$ is an arbitrary parameter. Then, $\widetilde{P}_{3}$ is transformed to the canonical symplectic matrix. In the coordinates $\left(q_{1}, p_{1}, q_{2}, p_{2}\right)$, the above two isomonodromic deformation equations are Hamiltonian systems. The Hamiltonian functions are obtained by deleting $W_{2}, W_{4}, W_{6}$ from $\widetilde{\psi}_{4}$ and $\widetilde{\psi}_{5}$ by using the above relations, and by changing to Darboux's coordinates. It is easy to verify that if we set $\alpha_{10}=\alpha_{5}^{2}$, then two functions become polynomials. In this manner, we obtain Hamiltonian functions (1.8) of $\left(\mathrm{P}_{\mathrm{II}-1}\right)_{2}$ given in Section 1.

(iii) Consider the vector fields $\widetilde{P}_{2} d \widetilde{\psi}_{3}$ and $\widetilde{P}_{2} d \widetilde{\psi}_{4}$. For the Poisson tensor $\widetilde{P}_{2}$, a symplectic leaf is defined by the level surface $\left\{\widetilde{\psi}_{j}=\right.$ const, $\left.j=1,2,5,6\right\}$. In this case, we cannot find an integer $l$ and a parameter $\alpha^{\prime}$ such that the condition

$$
\frac{\partial \widetilde{X}_{\lambda}}{\partial \alpha^{\prime}}=\lambda^{l} \frac{\partial A_{2}}{\partial \lambda}
$$

holds. Hence, we impose only one condition

$$
\frac{\partial \widetilde{X}_{\lambda}}{\partial \alpha}=\lambda^{2} \frac{\partial A_{1}}{\partial \lambda}=\lambda^{2}\left(\begin{array}{ll}
0 & 0 \\
1 & 0
\end{array}\right) .
$$

For it, $W_{2}$ in $\tilde{X}_{\lambda}$ has to include a parameter $\alpha$. To this end, take the symplectic leaf

$$
S=\left\{\widetilde{\psi}_{1}=1, \widetilde{\psi}_{2}=\alpha_{2}, \widetilde{\psi}_{5}=\alpha_{8}, \widetilde{\psi}_{6}=\alpha_{10}\right\}
$$

This is rearranged as

$$
W_{2}=\alpha_{2}-V_{2}, \quad W_{4}=\left(\alpha_{8}-2 U_{3} U_{5}-V_{2} W_{6}\right) / V_{4}, \quad W_{6}=\left(\alpha_{10}-U_{5}^{2}\right) / V_{4} .
$$

Substituting them into $\widetilde{X}_{\lambda}$ and $A_{1}$, we can verify the condition (4.11) with $\alpha=\alpha_{2}$. By replacing $\alpha_{2}$ by $t$, we obtain the isomonodromic deformation equation

$$
\frac{\partial L_{\lambda}}{\partial t}=\left[A_{1}, L_{\lambda}\right]+\frac{\partial A_{1}}{\partial \lambda}
$$

The Poisson tensor $\widetilde{P}_{2}$ on the symplectic leaf with coordinates $\left(U_{3}, V_{2}, U_{5}, V_{4}\right)$ is given by

$$
\widetilde{P}_{2}=\left(\begin{array}{cccc}
0 & -V_{2} & 0 & -V_{4} \\
V_{2} & 0 & V_{4} & 0 \\
0 & -V_{4} & 0 & 0 \\
V_{4} & 0 & 0 & 0
\end{array}\right)
$$


To change to Darboux's coordinates, put

$$
\left(U_{3}, V_{2}, U_{5}, V_{4}\right)=\left(p_{1} q_{1}+p_{2} q_{2}-\beta_{3}, p_{2}, p_{1} q_{2}-\beta_{5}, p_{1}\right),
$$

where $\beta_{3}$ and $\beta_{5}$ are arbitrary parameters. Then, $\widetilde{P}_{2}$ is transformed to the canonical symplectic matrix. In the coordinates $\left(q_{1}, p_{1}, q_{2}, p_{2}\right)$, the above isomonodromic deformation equation is

a Hamiltonian system. The Hamiltonian function is obtained by deleting $W_{2}, W_{4}, W_{6}$ from $\widetilde{\psi}_{3}$ by using the above relations, and by changing to Darboux's coordinates. It is easy to verify that if we set $\alpha_{10}=\beta_{5}^{2}$ and $\alpha_{8}=2 \beta_{3} \beta_{5}$, then $\widetilde{\psi}_{3}$ written in the coordinates $\left(q_{1}, p_{1}, q_{2}, p_{2}\right)$ becomes a polynomial. This procedure yields the Hamiltonian function (1.12) given in Section 1.

\section{4-dimensional Painlevé equations: $\mathfrak{g}=\mathfrak{s o}_{5}, n=1$}

According to [8], we use the following representation for the Lie algebra $\mathfrak{g} \simeq \mathfrak{s o}_{5}$ of type $\mathrm{B}_{2}$

$$
X_{i}=\left(\begin{array}{ccccc}
p_{i} & q_{i} & r_{i} & s_{i} & 0 \\
t_{i} & u_{i} & v_{i} & 0 & s_{i} \\
w_{i} & x_{i} & 0 & v_{i} & -r_{i} \\
y_{i} & 0 & x_{i} & -u_{i} & q_{i} \\
0 & y_{i} & -w_{i} & t_{i} & -p_{i}
\end{array}\right) .
$$

Consider the Lie algebra $\mathfrak{g}_{1}=\left\{X_{\lambda}=\lambda X_{0}+X_{1} \mid X_{i} \in \mathfrak{g} \simeq \mathfrak{s o}_{5}\right\}$. For the definition of $\mathfrak{g}_{1}^{0}$, we only consider the following case

$$
X_{0}=\left(\begin{array}{lllll}
0 & 0 & 0 & 0 & 0 \\
1 & 0 & 0 & 0 & 0 \\
0 & 1 & 0 & 0 & 0 \\
0 & 0 & 1 & 0 & 0 \\
0 & 0 & 0 & 1 & 0
\end{array}\right)
$$

(i.e., $x_{0}=t_{0}=1$ and zeros otherwise). The purpose in this section is to derive the Hamiltonian (1.13) for Cosgrove's equation. The other choice of $X_{0}$ may yield different Painlevé systems. Note that $n=1, \operatorname{rank}(\mathfrak{g})=h=2, m_{1}=1$ and $m_{2}=3$. We have the following functions

$$
\begin{aligned}
\psi_{1,1}= & -2 q_{1}-2 v_{1} \\
\psi_{1,2}= & -2 q_{1} t_{1}-u_{1}^{2}-2 v_{1} x_{1}-2 s_{1} y_{1}, \\
\psi_{2,1}= & -2 s_{1} \\
\psi_{2,2}= & q_{1}^{2}-2 s_{1} t_{1}+2 q_{1} v_{1}-4 s_{1} x_{1}, \\
\psi_{2,3}= & 2 q_{1}^{2} t_{1}+2 q_{1} t_{1} v_{1}+2 s_{1} u_{1} w_{1}-4 s_{1} t_{1} x_{1}+2 q_{1} v_{1} x_{1}-2 s_{1} x_{1}^{2}-2 q_{1} s_{1} y_{1}+2 s_{1} v_{1} y_{1}, \\
\psi_{2,4}= & q_{1}^{2} t_{1}^{2}-2 q_{1} u_{1} v_{1} w_{1}+2 q_{1} s_{1} w_{1}^{2}+2 q_{1} t_{1} v_{1} x_{1}+2 s_{1} u_{1} w_{1} x_{1} \\
& -2 s_{1} t_{1} x_{1}^{2}-2 q_{1} s_{1} t_{1} y_{1}-2 q_{1} v_{1}^{2} y_{1}+2 s_{1} v_{1} x_{1} y_{1}+s_{1}^{2} y_{1}^{2},
\end{aligned}
$$

which are coefficients of the characteristic polynomial $\operatorname{det}\left(\mu-X_{\lambda}\right)$.

We solve the differential equations for the two dimensional distribution $D$ as functions of $\left(p_{1}, r_{1}\right)$ with the initial condition $\left(q_{1}, s_{1}, t_{1}, u_{1}, v_{1}, w_{1}, x_{1}, y_{1}\right)=(Q, S, T, U, V, W, X, Y)$ at $\left(p_{1}, r_{1}\right)=(0,0)$. The expressions of solutions are too long and omitted here. These solutions define a coordinate transformation

$$
\left(p_{1}, r_{1}, q_{1}, s_{1}, t_{1}, u_{1}, v_{1}, w_{1}, x_{1}, y_{1}\right) \mapsto\left(p_{1}, r_{1}, Q, S, T, U, V, W, X, Y\right) .
$$


In the new coordinates, integral manifolds of the distribution $D$ are plains which are parallel to the $\left(p_{1}, r_{1}\right)$-plain. In particular, the subset $\left\{p_{1}=r_{1}=0\right\} \subset \mathfrak{g}_{1}^{0}$ gives the realization of the orbit space $\mathfrak{g}_{1}^{0} / D$ as a submanifold and $(Q, S, T, U, V, W, X, Y)$ provides a global coordinate system of $\mathfrak{g}_{1}^{0} / D$.

At this stage, we have on $\mathfrak{g}_{1}^{0} / D$

$$
\begin{aligned}
& \widetilde{\psi}_{1,1}=-2 Q-2 V \\
& \widetilde{\psi}_{1,2}=-2 Q T-U^{2}-2 V X-2 S Y, \\
& \widetilde{\psi}_{2,1}=-2 S \\
& \widetilde{\psi}_{2,2}=Q^{2}-2 S T+2 Q V-4 S X, \\
& \widetilde{\psi}_{2,3}=2 Q^{2} T+2 Q T V+2 S U W-4 S T X+2 Q V X-2 S X^{2}-2 Q S Y+2 S V Y,
\end{aligned}
$$

and the vector field $\widetilde{P}_{2} d \widetilde{\psi}_{2,3}$, whose Casimir functions are $\widetilde{\psi}_{1,1}, \widetilde{\psi}_{1,2}, \widetilde{\psi}_{2,1}$ and $\widetilde{\psi}_{2,2}$. The corresponding differential equation is expressed in Lax form as $d \widetilde{X}_{\lambda} / d t=\left[A_{\lambda}, \widetilde{X}_{\lambda}\right]$, where

$$
\begin{aligned}
& X_{\lambda}=\lambda\left(\begin{array}{ccccc}
0 & 0 & 0 & 0 & 0 \\
1 & 0 & 0 & 0 & 0 \\
0 & 1 & 0 & 0 & 0 \\
0 & 0 & 1 & 0 & 0 \\
0 & 0 & 0 & 1 & 0
\end{array}\right)+\left(\begin{array}{ccccc}
0 & Q & 0 & S & 0 \\
T & U & V & 0 & S \\
W & X & 0 & V & 0 \\
Y & 0 & X & -U & Q \\
0 & Y & -W & T & 0
\end{array}\right), \\
& A_{\lambda}=\lambda \nabla_{1} \widetilde{\psi}_{2,1}+\nabla_{1} \widetilde{\psi}_{2,2}+(V-Q) \nabla_{1} \widetilde{\psi}_{1,1}+\frac{2}{S}\left(Q^{2}-S X\right) \nabla_{1} \widetilde{\psi}_{2,1}, \\
& \nabla_{1} \widetilde{\psi}_{2,1}=\left(\begin{array}{ccccc}
0 & 0 & 0 & 0 & 0 \\
0 & 0 & 0 & 0 & 0 \\
0 & 0 & 0 & 0 & 0 \\
-2 & 0 & 0 & 0 & 0 \\
0 & -2 & 0 & 0 & 0
\end{array}\right), \quad \nabla_{1} \widetilde{\psi}_{1,1}=\left(\begin{array}{ccccc}
0 & 0 & 0 & 0 & 0 \\
-2 & 0 & 0 & 0 & 0 \\
0 & -2 & 0 & 0 & 0 \\
0 & 0 & -2 & 0 & 0 \\
0 & 0 & 0 & -2 & 0
\end{array}\right), \\
& \nabla_{1} \widetilde{\psi}_{2,2}=\left(\begin{array}{ccccc}
0 & 0 \\
2(Q+V) & -2 S & 0 & 0 & 0 \\
-2 U & 2 Q & 0 & -4 S & 0 \\
-2(T+2 X) & 0 & 2 Q & 0 & -2 S \\
0 & -2(T+2 X) & 2 U & 2(Q+V) & 0
\end{array}\right) .
\end{aligned}
$$

The next purpose is to restrict the vector field on a symplectic leaf. For the Poisson tensor $\widetilde{P}_{2}$, a symplectic leaf is defined by the level surface $\left\{\widetilde{\psi}_{i, j}=\right.$ const, $\left.i, j=1,2\right\}$. In order for the condition

$$
\frac{\partial \widetilde{X}_{\lambda}}{\partial \alpha}=\frac{\partial A_{\lambda}}{\partial \lambda}=\left(\begin{array}{ccccc}
0 & 0 & 0 & 0 & 0 \\
0 & 0 & 0 & 0 & 0 \\
0 & 0 & 0 & 0 & 0 \\
-2 & 0 & 0 & 0 & 0 \\
0 & -2 & 0 & 0 & 0
\end{array}\right)
$$

to be satisfied, we find that $Y$ in $\widetilde{X}_{\lambda}$ has to include a parameter $\alpha$ which will be replaced by $t$ later. For this purpose, we take the symplectic leaf

$$
S=\left\{\widetilde{\psi}_{2,1}=-2, \widetilde{\psi}_{1,1}=-2 \alpha_{2}, \widetilde{\psi}_{2,2}=-2 \alpha_{4}+\alpha_{2}^{2}, \widetilde{\psi}_{1,2}=4 \alpha_{6}-2 \alpha_{2} \alpha_{4}\right\} .
$$

This is rewritten as

$$
\begin{aligned}
& S=1, \quad Q=\alpha_{2}-V, \quad T=\alpha_{4}-2 X-\frac{1}{2} V^{2}, \\
& Y=-2 \alpha_{6}-\frac{1}{2} U^{2}-\frac{1}{2} V^{3}-3 V X+\frac{\alpha_{2}}{2} V^{2}+2 \alpha_{2} X+\alpha_{4} V .
\end{aligned}
$$


Substituting them into $\widetilde{X}_{\lambda}$ and $A_{\lambda}$, it turns out that the condition (5.1) is satisfied with $\alpha=\alpha_{6}$. Finally, by replacing $\alpha_{6}$ by $t$, we obtain the isomonodromic deformation equation (2.4). The Poisson tensor $\widetilde{P}_{2}$ written with respect to the coordinates $(U, V, W, X)$ is already in the canonical symplectic matrix. Thus, the isomonodromic deformation equation is a Hamiltonian system with the Hamiltonian function $\widetilde{\psi}_{2,3}$ written in the coordinate system $(U, V, W, X)$. Since this expression is too complicated, we further introduce the symplectic transformation

$$
\begin{array}{r}
(U, V, W, X)=\left(p_{2}, q_{1}+\frac{13}{18} \alpha_{2}, p_{1}+\frac{4}{13} p_{2}\left(q_{1}+\frac{13}{18} \alpha_{2}\right),\right. \\
\left.q_{2}+\frac{1}{3} \alpha_{4}+\frac{7}{108} \alpha_{2}^{2}-\frac{2}{13}\left(q_{1}+\frac{13}{18} \alpha_{2}\right)^{2}\right) .
\end{array}
$$

Then, the Hamiltonian function (1.15), which is equivalent to (1.13), is obtained.

\section{References}

[1] Adler M., van Moerbeke P., Vanhaecke P., Algebraic integrability, Painlevé geometry and Lie algebras, Ergebnisse der Mathematik und ihrer Grenzgebiete. 3. Folge. A Series of Modern Surveys in Mathematics, Vol. 47, Springer-Verlag, Berlin, 2004.

[2] Chiba H., The first, second and fourth Painlevé equations on weighted projective spaces, J. Differential Equations 260 (2016), 1263-1313, arXiv:1311.1877.

[3] Chiba H., The third, fifth and sixth Painlevé equations on weighted projective spaces, SIGMA 12 (2016), 019, 22 pages, arXiv:1506.00444.

[4] Chiba H., Painlevé equations and weights, submitted.

[5] Clarkson P.A., Joshi N., Mazzocco M., The Lax pair for the mKdV hierarchy, in Théories asymptotiques et équations de Painlevé, Sémin. Congr., Vol. 14, Soc. Math. France, Paris, 2006, 53-64.

[6] Clarkson P.A., Joshi N., Pickering A., Bäcklund transformations for the second Painlevé hierarchy: a modified truncation approach, Inverse Problems 15 (1999), 175-187, solv-int/9811014.

[7] Cosgrove C.M., Chazy classes IX-XI of third-order differential equations, Stud. Appl. Math. 104 (2000), $171-228$

[8] Drinfel'd V.G., Sokolov V.V., Lie algebras and equations of Korteweg-de Vries type, J. Math. Sci. 30 (1985), 1975-2036.

[9] Falqui G., Magri F., Pedroni M., Zubelli J.P., A bi-Hamiltonian theory for stationary KDV flows and their separability, Regul. Chaotic Dyn. 5 (2000), 33-52, nlin.SI/0003020.

[10] Gordoa P.R., Joshi N., Pickering A., On a generalized $2+1$ dispersive water wave hierarchy, Publ. Res. Inst. Math. Sci. 37 (2001), 327-347.

[11] Koike T., On new expressions of the Painlevé hierarchies, in Algebraic Analysis and the Exact WKB Analysis for Systems of Differential Equations, RIMS Kôkyûroku Bessatsu, Vol. B5, Res. Inst. Math. Sci. (RIMS), Kyoto, 2008, 153-198.

[12] Kudryashov N.A., The first and second Painlevé equations of higher order and some relations between them, Phys. Lett. A 224 (1997), 353-360.

[13] Levin A.M., Olshanetsky M.A., Painlevé-Calogero correspondence, in Calogero-Moser-Sutherland Models (Montréal, QC, 1997), CRM Ser. Math. Phys., Springer, New York, 2000, 313-332.

[14] Magnano G., Magri F., Poisson-Nijenhuis structures and Sato hierarchy, Rev. Math. Phys. 3 (1991), 403466.

[15] Magri F., Casati P., Falqui G., Pedroni M., Eight lectures on integrable systems, in Integrability of Nonlinear Systems (Pondicherry, 1996), Lecture Notes in Phys., Vol. 495, Springer, Berlin, 1997, 256-296.

[16] Magri F., Falqui G., Pedroni M., The method of Poisson pairs in the theory of nonlinear PDEs, in Direct and inverse methods in nonlinear evolution equations, Lecture Notes in Phys., Vol. 632, Springer, Berlin, 2003, 85-136, nlin.SI/0002009.

[17] Nakamura A., Autonomous limit of 4-dimensional Painlevé-type equations and degeneration of curves of genus two, arXiv:1505.00885.

[18] Shimomura S., A certain expression of the first Painlevé hierarchy, Proc. Japan Acad. Ser. A Math. Sci. 80 (2004), 105-109. 